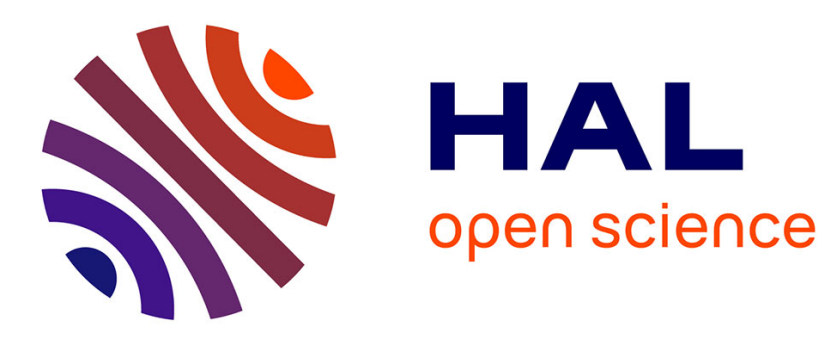

\title{
Kinetic-magnetic energy exchanges in rotating magnetohydrodynamic turbulence
}

F. S. Baklouti, A. Khlifi, A. Salhi, F. Godeferd, C. Cambon, T. Lehner

\section{To cite this version:}

F. S. Baklouti, A. Khlifi, A. Salhi, F. Godeferd, C. Cambon, et al.. Kinetic-magnetic energy exchanges in rotating magnetohydrodynamic turbulence. Journal of Turbulence, 2019, 20 (4), pp.263284. 10.1080/14685248.2019.1623897 . hal-02906455

\section{HAL Id: hal-02906455 \\ https://hal.science/hal-02906455}

Submitted on 18 Nov 2020

HAL is a multi-disciplinary open access archive for the deposit and dissemination of scientific research documents, whether they are published or not. The documents may come from teaching and research institutions in France or abroad, or from public or private research centers.
L'archive ouverte pluridisciplinaire HAL, est destinée au dépôt et à la diffusion de documents scientifiques de niveau recherche, publiés ou non, émanant des établissements d'enseignement et de recherche français ou étrangers, des laboratoires publics ou privés. 


\title{
Kinetic-magnetic energy exchanges in rotating magnetohydrodynamic turbulence
}

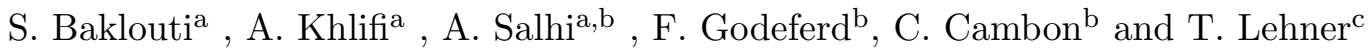 \\ ${ }^{a}$ Département de Physique, Faculté des Sciences de Tunis, Université Tunis El Manar, Tunis, \\ Tunisia; \\ ${ }^{\text {b} U n i v e r s i t e ́ ~ d e ~ L y o n, ~ L a b o r a t o i r e ~ d e ~ M e ́ c a n i q u e ~ d e s ~ F l u i d e s ~ e t ~ d ' A c o u s t i q u e, ~ U M R ~ 5509, ~}$ \\ Ecole Centrale de Lyon, CNRS, UCBL, INSA F-69134 Ecully Cedex, France; \\ ${ }^{\mathrm{c}}$ LUTH, UMR 8102 CNRS, Observatoire de Paris-Meudon, 5 place de Janssen, F-92195 \\ Meudon, France.
}

\section{ARTICLE HISTORY}

Compiled May 17, 2019

\begin{abstract}
We use direct numerical simulations to study the dynamics of incompressible homogeneous turbulence subjected to a uniform magnetic field $\boldsymbol{B}$ (the Alfvén velocity) in a rotating frame with rotation vector $\boldsymbol{\Omega}$. We consider two cases: $\boldsymbol{\Omega} \| \boldsymbol{B}$ and $\boldsymbol{\Omega} \perp \boldsymbol{B}$. The initial flow state is homogeneous isotropic hydrodynamic turbulence with kinetic Reynolds number $\operatorname{Re}=u_{l} l / \nu \simeq 170$. The magnetic Prandtl number is $\mathrm{Pm}=\nu / \eta=1$ and the Elsasser number $\Lambda=B^{2} /(2 \Omega \eta)=0.5,0.9$ or 2. Both for $\boldsymbol{\Omega} \| \boldsymbol{B}$ and $\boldsymbol{\Omega} \perp \boldsymbol{B}$, the total (kinetic + magnetic) energy $E$ decays as $\sim t^{-5 / 7}$ for $\Lambda=0.5$ and 0.9 , and as $\sim t^{-6 / 7}$ for $\Lambda=2$. In the spectral range $2<k<20$, the radial (spherically averaged) spectrum of kinetic energy scales as $\sim k^{-p}$ where the index $p$ increases with time $(2 \leq p \leq 4.2)$, or equivalently, with the interaction parameter $\mathrm{N}=B^{2} l /\left(\eta u_{l}\right)$. This time-dependent scaling is similar to that observed in quasi-static MHD. The two rotating MHD flow cases differ mainly in how kinetic and magnetic fluctuations exchange energy, with a mechanism mostly driven by the dynamics of the spectral buffer layer around $k_{\|}^{\Omega}=|\boldsymbol{\Omega} \cdot \boldsymbol{k}| / \Omega \approx 0$. At $k_{\|}^{\Omega}=0$, both the frequencies of inertial and Alfvén waves vanish when $\boldsymbol{\Omega} \| \boldsymbol{B}$, but only the frequency of inertial waves vanishes for the case when $\boldsymbol{\Omega} \perp \boldsymbol{B}$. When $\boldsymbol{\Omega} \| \boldsymbol{B}$, rotation results in an increased reduction of magnetic fluctuations generation. In terms of anisotropy, we show that the elongated structures occurring in rapidly non-magnetized rotating flows are distorted or inhibited for $\boldsymbol{\Omega} \perp \boldsymbol{B}$, and their intensity is weakened for $\Omega \| B$.

Keywords: Direct numerical simulations, Homogeneous rotating magnetohydrodynamic turbulence, Kinetic-magnetic energy exchanges, Inertial waves, Alfvén waves.
\end{abstract}

\section{Introduction}

Magnetohydrodnamic (MHD) flows are ubiquitous in several geophysical and astrophysical systems (the Earth atmosphere and outer core, magnetized planets such as Jupiter or Saturn, Solar wind, Solar interior and atmosphere, accretion disks,...). Most of these flows are turbulent (i.e. they are characterized by large kinetic and magnetic Reynolds numbers, Re $\gg 1, R m \gg 1[1,2]$ ) and often subject to rotation (denoting by $\boldsymbol{\Omega}$ the rotation vector), mean magnetic field (denoting by $\boldsymbol{B}$ the Alfvén velocity) or both [3]. The kinetic and magnetic Reynolds numbers are defined as $\operatorname{Re}=l u_{l} / \nu$ and $\mathrm{Rm}=l u_{l} / \eta$ where $l$ is a characteristic length scale, $u_{l}$ is the associated velocity (thus the ratio $l / u_{l}$ represents the nonlinear eddy turnover time), $\nu$ is the kinematic viscosity, and $\eta$ is the magnetic diffusivity.

CONTACT A. Salhi. Email: lazizsalhi@yahoo.fr 
For magnetized planets like Earth, Jupiter or Saturn, the rotation and magnetic axes are close and even almost perfectly aligned for Saturn $[4,5]$. In contrast, fastspinning highly-magnetized neutron stars (the so-called millisecond magnetars) with predominantly toroidal magnetic fields might flip over until the magnetic and rotation axes are orthogonal [6]. Also, in the Earth's core, the strong differential rotation between the inner core and the mantle causes the magnetic field to be mainly toroidal, thus locally perpendicular to the rotation axis [3].

The study of the combined effects of the Coriolis and Lorentz forces on the dynamics of incompressible homogeneous MHD turbulence is relevant for these geophysical and astrophysical flows [5]. The incompressible MHD equations, relevant for studying the dynamics of such flows, show that the inviscid linear regime is characterized by propagating magnetic-Coriolis waves with frequency $[4,5,7,8]$

$$
\omega_{f, s}=\frac{1}{2}\left(\sqrt{\omega_{R}^{2}+4 \omega_{A}^{2}} \pm \omega_{R}\right)
$$

where $\omega_{R}=2|\boldsymbol{\Omega} \cdot \boldsymbol{k}| / k$ is the inertial waves frequency and $\omega_{A}=|\boldsymbol{B} \cdot \boldsymbol{k}|$ is the Alfvén waves frequency, and $\boldsymbol{k}$ is the wave vector of modulus $k$. The relation dispersion (1) describes both fast (subscript $f$ ) and slow (subscript $s$ ) magnetic Coriolis (MC) waves even if $\boldsymbol{\Omega}$ and $\boldsymbol{B}$ are not aligned [8].

Turbulent motions and waves propagation interplay with two different regimes: the regime in which the linear terms dominate over the nonlinear terms (or equivalently, when the waves period remains much smaller than the eddy-turn-over time), is known as weak wave turbulence; otherwise the turbulence is called strong [10-14,48]. Note that for the non rotating MHD case, the mean magnetic field induces the development of flow anisotropy with $\boldsymbol{B}$ the preferential direction [15], such that for intense $\boldsymbol{B}$ the flow can become quasi-two-dimensional, with very weak variations along $\boldsymbol{B}$ [16-18]. Similarly, in rapidly rotating turbulence of non conducting fluid, the emergence of large-scale vortices characterizes a quasi-two-dimensional state perpendicular to fixed rotation axis [19], and at increasing rotation rate, energy accumulates in the vicinity of the so-called spectral buffer layer around $k_{\|}^{\Omega} \equiv|\boldsymbol{\Omega} \cdot \boldsymbol{k}| / \Omega=0[20,21]$.

For rotating MHD turbulence, the configuration of mean magnetic field aligned with rotation (hereinafter referred to as parallel case) has been extensively addressed $[5,7,8,22]$. In the laboratory experiment of a magnetized turbulent Taylor-Couette flow of liquid metal by Nornberg et al. [23], the combined fast and slow MC waves were clearly identified, but the observed slow MC wave are damped. Obviously, in that case, the interaction of the background shear induced by the differential rotation with MC waves can generate the magnetorotational instability [23-25]. The dispersion relation for the MC waves in the parallel case reduces to $\omega_{f, s}=\left(\Omega k_{\|}^{\Omega} / k\right)\left(\sqrt{1+\mathcal{L}_{k}^{2}} \pm 1\right)$ where $\mathcal{L}_{k}=B k / \Omega$ is the local Lehnert number [26]. This indicates that in the largescale limit $\left(\mathcal{L}_{k} \ll 1\right)$, the fast and slow waves respectively tend to the inertial and magnetostrophic waves [27], whereas in the small-scale limit pure Alfvén waves are recovered [5]. The weak wave turbulence theory by Galtier [5] in the limit of small Rossby and Ekman numbers $\left(\mathrm{Ro}=u_{l} /(2 \Omega l) \ll 1\right.$ and $\left.\mathrm{E}=\nu /\left(2 \Omega l^{2}\right) \ll 1\right)$ show that inertial wave turbulence is asymptotically dominated by the kinetic energy whereas the magnetostrophic wave turbulence is dominated by the magnetic energy. The nonlinear dynamics is mainly anisotropic with a stronger transfer perpendicular than parallel to the rotating axis [5].

Direct numerical simulations (DNS) by Favier et al. [7] of the parallel case at large magnetic Reynolds number $(\mathrm{Rm}=\mathrm{Re} \sim 200)$, moderate interaction parameter $(\mathrm{N}=$ $\left.B^{2} l /(\eta u) \sim 12\right)$ and small Rossby number regime $(\mathrm{Ro}<1)$ show that the equipartition between kinetic and magnetic energy due to Alfvén waves [28] is broken by inertial 
waves. The energy is concentrated in the modes perpendicular to the rotation axis, which corresponds to the two-dimensional manifold in physical space.

Note that many of the features of large-scale dynamics in rotating MHD turbulence can be explained by linear analysis $[7,8]$. For instance, the linear theory solution [8] indicates that, at large scales $\left(\mathcal{L}_{k} \ll 1\right)$, the Alfvén energy ratio (kinetic over magnetic) scales as $k^{-2}$ for the parallel case. In contrast, in the perpendicular case, the Alfvén energy ratio scales as $k^{-1}$. Therefore, at large scales, there is more energy exchange between velocity $\boldsymbol{u}(\boldsymbol{x}, t)$ and magnetic $\boldsymbol{b}(\boldsymbol{x}, t)$ fluctuations in the parallel case than in the perpendicular one. At small scales $\left(\mathcal{L}_{k} \gg 1\right)$, the Alfvén energy ratio approaches unity for both cases.

In the present study, we perform DNS for an initially isotropic homogeneous turbulence subject to a uniform magnetic field in a frame rotating about an axis that is either parallel or perpendicular to the imposed external magnetic field. We consider freely decaying turbulence without forcing. Our main purpose is to study the effects of rotation on the exchanges between kinetic and magnetic energies. We analyze the development of energies and associated radial (spherically averaged) spectra that provide information about the distribution of energy at different scales. We attempt to explain why there is more energy exchanges between kinetic and magnetic components in the case where the imposed magnetic field is perpendicular to the rotation axis than in the parallel case. The role of the $k_{\|}^{\Omega}=0$ mode on the energy exchanges is also addressed. The paper is organized as follows. The model equations in physical and spectral spaces are presented in section 2. Section 3 is devoted to the discussion of the DNS results: decay powerlaw for total (kinetic + magnetic) energy and dissipation rate, radial spectra, two-dimensional (2D) energies ,especially those for $k_{\|}^{\Omega}=0$, and anisotropy. Our concluding remarks are presented in section 4 .

\section{Model Equations}

\subsection{Incompressible MHD equations}

We consider homogeneous turbulence of incompressible electrically conducting fluid under solid rotation $\boldsymbol{\Omega}$ and in the presence of a uniform background magnetic field $\boldsymbol{B}$. In the following, mean and fluctuating magnetic fields are scaled using Alfvén velocity units i.e. they are divided by $\sqrt{\rho_{0} \mu_{0}}$ where $\rho_{0}$ and $\mu_{0}$ are the constant density and the magnetic permeability. Incompressible MHD equations provide the simplest theoretical framework for studying large-scale turbulent motion of an electrically conducting fluid. Accordingly, the equations for the fluctuating velocity $\boldsymbol{u}$ and magnetic field $\boldsymbol{b}$ are [29]

$$
\begin{aligned}
\left(\partial_{t}+\boldsymbol{u} \cdot \nabla\right) \boldsymbol{u} & =-\nabla p-2 \boldsymbol{\Omega} \times \boldsymbol{u}+((\boldsymbol{B}+\boldsymbol{b}) \cdot \nabla) \boldsymbol{b}+\nu \nabla^{2} \boldsymbol{u}, \\
\left(\partial_{t}+\boldsymbol{u} \cdot \nabla\right) \boldsymbol{b} & =((\boldsymbol{B}+\boldsymbol{b}) \cdot \nabla) \boldsymbol{u}+\eta \nabla^{2} \boldsymbol{b}, \\
\nabla \cdot \boldsymbol{u} & =0, \nabla \cdot \boldsymbol{b}=0,
\end{aligned}
$$

where $p$ is the fluctuating pressure (including the magnetic part), $\nu$ is the kinematic viscosity and $\eta$ is the magnetic diffusivity. We choose $\nu=\eta$, so that the magnetic Prandtl number is $\mathrm{Pm}=\eta / \nu=1$. In the momentum conservation equation (2a), $(\boldsymbol{u} \cdot \nabla) \boldsymbol{u}$ is the inertial term, and $-2 \boldsymbol{\Omega} \times \boldsymbol{u}$ and $((\boldsymbol{B}+\boldsymbol{b}) \cdot \nabla) \boldsymbol{b}$ are the Coriolis and Lorentz forces respectively. In the induction equation $(2 \mathrm{~b}),(\boldsymbol{u} \cdot \nabla) \boldsymbol{b}$ and $((\boldsymbol{B}+\boldsymbol{b}) \cdot \nabla) \boldsymbol{u}$ respectively represent the advection and stretching of magnetic field.

Assuming that the turbulence is statistically homogeneous, the resulting equations for the kinetic and magnetic energies per unit mass $E^{(\kappa)}=\frac{1}{2}\left\langle u_{i} u_{i}\right\rangle$ and $E^{(m)}=\frac{1}{2}\left\langle b_{i} b_{i}\right\rangle$ 
— where $\langle\cdot\rangle$ denotes ensemble (statistical) averaging — are

$$
\begin{aligned}
\partial_{t} E^{(\kappa)} & =-\varepsilon^{(\kappa)}-\mathcal{F}^{(\kappa m)} \\
\partial_{t} E^{(m)} & =-\varepsilon^{(m)}+\mathcal{F}^{(\kappa m)}
\end{aligned}
$$

where $\varepsilon^{(\kappa)}=\nu\left\langle\partial_{x_{j}} u_{i} \partial_{x_{j}} u_{i}\right\rangle$ and $\varepsilon^{(m)}=\eta\left\langle\partial_{x_{j}} b_{i} \partial_{x_{j}} b_{i}\right\rangle$ are respectively the viscous and magnetic (or Joule) dissipation rates per unit mass, and

$$
\mathcal{F}^{(\kappa m)}=B_{j}\left\langle b_{i} \partial_{x_{j}} u_{i}\right\rangle=-B_{j}\left\langle u_{i} \partial_{x_{j}} b_{i}\right\rangle
$$

represents the energy transfer between magnetic and kinetic energies due to the imposed magnetic field. Rotation produces no energy.

\subsection{Equations for Fourier amplitudes}

Fourier space representation is useful to quantify the energy contents at various scales. Therefore, we represent the fluctuating fields as the superposition of Fourier modes using the Fourier transforms

$$
[\boldsymbol{u}, p, \boldsymbol{b}]=\int_{\mathbb{R}^{3}}[\hat{\boldsymbol{u}}, \hat{p}, \hat{\boldsymbol{b}}] \exp \mathbf{i}(\boldsymbol{k} \cdot \boldsymbol{x}) d^{3} \boldsymbol{k}
$$

where $\boldsymbol{k}$ is the wave vector. It can be written as $\boldsymbol{k}=k_{1} \boldsymbol{e}_{1}+k_{2} \boldsymbol{e}_{2}+k_{3} \boldsymbol{e}_{3}$ in a polarspherical frame in which the vertical unit vector $\boldsymbol{e}_{3}=\Omega / \Omega$ aligns with the rotation vector. Thus, the equations for the Fourier coefficients $\hat{\boldsymbol{u}}(\boldsymbol{k}, t)$ and $\hat{\boldsymbol{b}}(\boldsymbol{k}, t)$ are

$$
\begin{aligned}
\dot{\hat{\boldsymbol{u}}}+2 \boldsymbol{\Omega} \times \hat{\boldsymbol{u}}-\mathbf{i}(\boldsymbol{k} \cdot \boldsymbol{B}) \hat{\boldsymbol{b}}+\mathbf{i} \hat{p}^{(\ell)} \boldsymbol{k}+\nu k^{2} \hat{\boldsymbol{u}} & =\boldsymbol{t}^{(\kappa)}, \\
\dot{\hat{\boldsymbol{b}}}-\mathbf{i}(\boldsymbol{k} \cdot \boldsymbol{B}) \hat{\boldsymbol{u}}+\eta k^{2} \hat{\boldsymbol{b}} & =\boldsymbol{t}^{(m)}, \\
\boldsymbol{k} \cdot \hat{\boldsymbol{u}}=0, \boldsymbol{k} \cdot \hat{\boldsymbol{b}} & =0,
\end{aligned}
$$

where $\hat{p}^{(\ell)}$ is the spectral counterpart of the linear part of pressure fluctuations. The two terms $\boldsymbol{t}^{(\kappa)}$ and $\boldsymbol{t}^{(m)}$ are bilinear operators for the kinetic and magnetic energy transfers, written as [30]

$$
\begin{aligned}
t_{\alpha}^{(\kappa)} & =-\mathbf{i} P_{\alpha \beta}(\boldsymbol{k}) k_{\gamma} \int_{\boldsymbol{p}+\boldsymbol{q}=\boldsymbol{k}} \hat{u}_{\beta}(\boldsymbol{p}, t) \hat{u}_{\gamma}(\boldsymbol{q}, t) d^{3} \boldsymbol{p}+\mathbf{i} P_{\alpha \beta}(\boldsymbol{k}) k_{\gamma} \int_{\boldsymbol{p}+\boldsymbol{q}=\boldsymbol{k}} \hat{b}_{\beta}(\boldsymbol{p}, t) \hat{b}_{\gamma}(\boldsymbol{q}, t) d^{3} \boldsymbol{p}, \\
t_{\alpha}^{(m)} & =-\mathbf{i} \delta_{\alpha \beta}(\boldsymbol{k}) k_{\gamma} \int_{\boldsymbol{p}+\boldsymbol{q}=\boldsymbol{k}} \hat{b}_{\beta}(\boldsymbol{p}, t) \hat{u}_{\gamma}(\boldsymbol{q}, t) d^{3} \boldsymbol{p}-\mathbf{i} \delta_{\alpha \beta}(\boldsymbol{k}) k_{\gamma} \int_{\boldsymbol{p}+\boldsymbol{q}=\boldsymbol{k}} \hat{b}_{\beta}(\boldsymbol{q}, t) \hat{u}_{\gamma}(\boldsymbol{p}, t) d^{3} \boldsymbol{p}
\end{aligned}
$$

where $P_{\alpha \beta}=\delta_{\alpha \beta}-k^{-2} k_{\alpha} k_{\beta}$ is the projection operator and $\delta_{\alpha \beta}$ is the Kronecker delta. The resulting equation for the spectral density of kinetic energy $e^{(\kappa)}=\frac{1}{2}\left\langle u_{i} u_{i}^{*}\right\rangle$ and that for the spectral magnetic energy density $e^{(m)}=\frac{1}{2}\left\langle b_{i} b_{i}^{*}\right\rangle$, where ${ }^{*}$ stands for complex conjugate, are

$$
\begin{gathered}
\partial_{t} e^{(\kappa)}+f^{(\kappa m)}+2 \nu k^{2} e^{(\kappa)}=T^{(\kappa \kappa)}+T^{(\kappa m)} \\
\partial_{t} e^{(m)}-f^{(\kappa m)}+2 \eta k^{2} e^{(m)}=T^{(m \kappa)}+T^{(m m)}
\end{gathered}
$$


where

$$
f^{(\kappa m)}=(\boldsymbol{k} \cdot \boldsymbol{B})\left\langle\Im\left(\hat{b}_{i} \hat{u}_{i}^{*}\right)\right\rangle
$$

again characterizes the energy exchange between the kinetic and magnetic components due to the background magnetic field $\boldsymbol{B}$. When $\boldsymbol{k} \perp \boldsymbol{B}=0$ there is no energy exchange due to the background magnetic field, i.e. $f^{(\kappa m)}=0$. This occurs for $k_{3}=0$ in the parallel case $\left(\boldsymbol{\Omega}=\Omega_{\boldsymbol{e}_{3}}, \boldsymbol{B}=B \boldsymbol{e}_{3}\right)$ or for $k_{1}=0$ in the perpendicular case $\left(\boldsymbol{\Omega}=\Omega \boldsymbol{e}_{3}, \boldsymbol{B}=\boldsymbol{B} \boldsymbol{e}_{1}\right) . T^{(\kappa \kappa)}, T^{(\kappa m)}, T^{(m \kappa)}$ and $T^{(m m)}$ are transfer-like terms that involve triple velocity and magnetic fluctuation correlations, and are such that $\int_{\mathbb{R}^{3}}\left(T^{(\kappa \kappa)}+T^{(\kappa m)}\right) d^{3} \boldsymbol{k}=0$ and $\int_{\mathbb{R}^{3}}\left(T^{(m \kappa)}+T^{(m m)}\right) d^{3} \boldsymbol{k}=0$. The energy exchange between kinetic and magnetic component due to nonlinear interactions is characterized by both terms $T^{(\kappa m)}$ and $T^{(m \kappa)}$.

In the direct numerical simulation approach used here, the fully nonlinear equations of motion (6) are solved using a modified version of the Fourier spectral code Snoopy by [31] as described in the following section.

\section{DNS Results and discussions}

\subsection{Initial conditions}

We initialize direct numerical simulations from homogeneous isotropic hydrodynamic turbulence generated by a separate simulation started from random initial conditions with the initial energy spectrum [32]

$$
S^{(\kappa)}(k)=C_{0} k^{4} \exp \left(-k^{2} / k_{p}^{2}\right)
$$

where $C_{0}$ is a normalization constant and $1 / k_{p}$ is a characteristic length scale $\left(k_{p}=6\right)$. $E^{(\kappa)}(0)=\int_{0}^{\infty} S^{(\kappa)}(k) d k$ is the initial kinetic energy. In order to let the turbulence develop a significant inertial zone as well as nonlinear transfer, an isotropic precomputation $[33,34]$ is done before applying the Coriolis and Lorentz forces. During this initial stage only, large-scale forcing is applied until a statistical steady state of classical Kolmogorov-like isotropic turbulence is reached. This forcing is applied for wavenumbers such that $1 \leq k \leq 4$. The domain is a periodic cube with edge length $L_{0}=2 \pi$ with $n_{0}=256$ grid points to a side. Aliasing errors are removed using the $2 / 3$ dealiasing rule and as a result the minimum and maximum wavenumbers are $k_{\min }=1$ and $k_{\max }=n_{0} / 3$ respectively. As in all DNS of decaying turbulence, one needs to minimize the effects of periodic boundary conditions that could affect the flow evolution, especially in anisotropic turbulence, as in rotation [11]. For that, we choose to let the flow evolve only if the largest integral length scale $\max \left|L_{j j}^{(\ell)}\right| \leq 0.25 L_{0}$ where

$$
L_{j j}^{(\ell)}=\frac{1}{\left\langle u_{j} u_{j}\right\rangle} \int_{0}^{\infty}\left\langle u_{j}(\boldsymbol{x}) u_{j}\left(\boldsymbol{x}+r \boldsymbol{e}_{\ell}\right)\right\rangle d r
$$

is the integral length scale in the $x_{\ell}(\ell=1,2,3)$ direction.

In our simulations, the initial characteristic length scale $l$ and the associated velocity $u_{l}$ are defined as

$$
u_{l}=u_{r m s}=\sqrt{\frac{2}{3} E^{(\kappa)}}, \quad l=\frac{u_{l}}{\pi} \frac{E}{\varepsilon},
$$

and chosen as $u_{l 0}=0.75$ and $l_{0}=0.58$ (see table 1 ), close to those used in the DNS 
by Favier et al. [7]. All our simulations (see table 2) are run up to $t^{+} \equiv t u_{l 0} / l_{0}=50$, i.e. fifty times the initial eddy turnover time, while in the study by Favier et al.[7], the final computational time is $t^{+} \simeq 5$.

Figure 1 shows the resulting precomputation kinetic energy spectrum $S^{(\kappa)}(k, 0)$. A $-5 / 3$ powerlaw scaling is observable in the range $3 \leq k \leq 20$ even for a resolution of $512^{3}$. Observable differences between the two resolution results $\left(n_{0}^{3}=256^{3}\right.$ and $n_{0}^{3}=512^{3}$ ) appear at high wavenumbers $k>10^{2}$. The inset of figure 1 shows the time evolution of the kinetic energy $E(t)$ in freely decaying isotropic hydrodynamic turbulence (IHT, run 0, see table 2) which is close to the Kolmogorov prediction $E(t) \sim t^{-10 / 7}$ (see e.g. [35]).

\begin{tabular}{ccccccccc}
\hline \hline$n_{0}$ & $u_{l}$ & $\varepsilon$ & $l$ & $\nu$ & $R_{e}$ & $R_{e \lambda}$ & $\gamma$ & $k_{\max \gamma}$ \\
\hline 256 & 0.75 & 0.35 & 0.58 & 0.0025 & 172 & 73 & 0.0145 & 1.241 \\
\hline 512 & 0.75 & 0.33 & 0.61 & 0.0025 & 182 & 76 & 0.0147 & 2.516
\end{tabular}

Table 1. Turbulence characteristics of the initial velocity field. The initial magnetic fluctuations are zero. Resolution: $n_{0}$; Rms value: $u_{l}=\sqrt{(2 / 3) E}$; Dissipation rate: $\varepsilon$; Characteristic length scale: $l=u_{l} E /(\pi \varepsilon)$; Viscosity: $\nu$, Reynolds number: Re $=u_{l} l / \nu$; Microscale Reynolds number: $\operatorname{Re}_{\lambda}=u_{l}^{2} \sqrt{15 /\left(\nu \varepsilon^{(\kappa)}\right)}$; Dissipation scale: $\gamma=\left(\nu^{3} / \varepsilon\right)^{\frac{1}{4}}$.

\begin{tabular}{lcccccccc}
\hline \hline Run & $\eta$ & Rem & $B$ & $N$ & $\Omega$ & Ro & $\operatorname{Ro}_{\omega}$ & $\Lambda$ \\
\hline 0 (decaying IHT) & - & - & - & - & - & - & - & - \\
1 (decaying MHD) & 0.0025 & 172 & 0.2 & 12 & 0 & 0 & 0 & $\infty$ \\
2 (decaying MHD) & 0.0025 & 172 & 1.5 & 675 & 0 & 0 & 0 & $\infty$ \\
3 (parallel case, $\boldsymbol{\Omega} \| \boldsymbol{B}$ ) & 0.0025 & 172 & 0.2 & 12 & 4 & 0.16 & 1.47 & 2.0 \\
4 (perpendicular case, $\boldsymbol{\Omega} \perp \boldsymbol{B})$ & 0.0025 & 172 & 0.2 & 12 & 4 & 0.16 & 1.47 & 2.0 \\
5 (parallel case, $\boldsymbol{\Omega} \| \boldsymbol{B}$ ) & 0.0025 & 172 & 0.2 & 12 & 9 & 0.07 & 0.65 & 0.9 \\
6 (perpendicular case, $\boldsymbol{\Omega} \perp \boldsymbol{B})$ & 0.0025 & 172 & 0.2 & 12 & 9 & 0.07 & 0.65 & 0.9 \\
7 (parallel case, $\boldsymbol{\Omega} \| \boldsymbol{B})$ & 0.0025 & 172 & 0.2 & 12 & 16 & 0.04 & 0.37 & 0.5 \\
8 (perpendicular case, $\boldsymbol{\Omega} \perp \boldsymbol{B})$ & 0.0025 & 172 & 0.2 & 12 & 16 & 0.04 & 0.37 & 0.5 \\
9 (rotating hydrodynamic case) & - & - & - & - & 9 & 0.07 & 0.65 & -
\end{tabular}

Table 2. Summary of the parameters for the different runs performed.

\subsection{Time evolution of energies}

In this part, we discuss the decay of total (kinetic + magnetic) energy $E=E^{(\kappa)}+E^{(m)}$ in freely decaying rotating MHD turbulence, which evolves according to $\partial_{t} E=-\varepsilon$. The total energy $E(t)$ is an inviscid invariant of MHD turbulence and as such deserves a specific discussion.

\subsubsection{Non-rotating MHD turbulence}

Previous studies have shown that MHD turbulence decay is slower than the decay of non-magnetic isotropic turbulence [36-39]. For instance, in non-helical isotropic MHD turbulence the total energy decays as $E(t) \sim t^{-1}$ [40,41]. Such predictions are at odds with Banerjee and Jedamzik [42], who predict larger decay rates for the kinetic and magnetic energies, closer to those of isotropic hydrodynamic turbulence (IHT). Recently, Briard and Gomez [43] have used a two-point spectral closure (the eddy-damped quasi-normal Markovian approximation, or EDQNM) to investigate the decay of isotropic MHD turbulence at very large Reynolds numbers. They found that the decay exponent of total energy matches the usual predictions for kinetic energy 


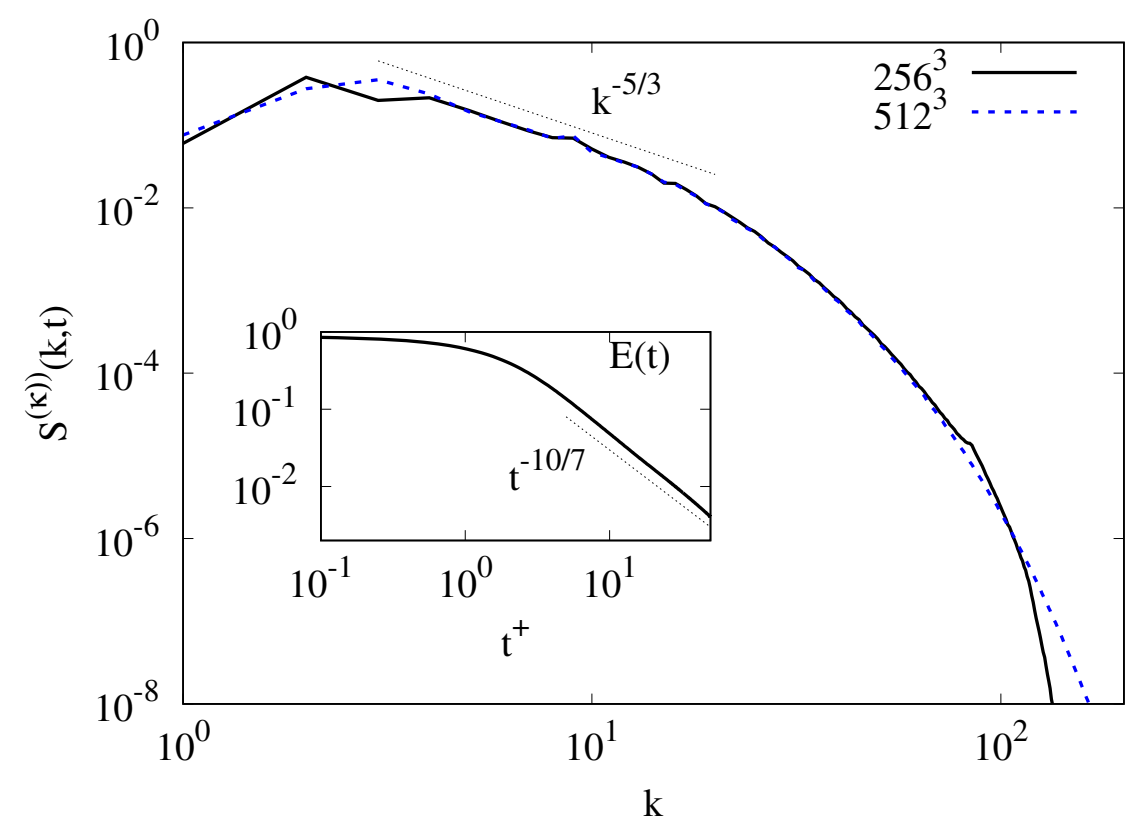

Figure 1. Resulting precomputation spectrum $S^{(\kappa)}(k, 0)$ of kinetic energy in forced isotropic hydrodynamic turbulence (THI) for the resolutions $n_{0}^{3}=256^{3}$ and $n_{0}^{3}=512^{3}$. The inset shows the time evolution of the kinetic energy $E(t)$ in freely decaying IHT (run 0, see table 2) which is close to the Kolmogorov prediction , $E(t) \sim t^{-10 / 7}$.

in IHT (i.e. $E(t) \propto t^{-10 / 7}$, see their equation 3.6), even at relatively low Reynolds numbers as well.

The role of a uniform magnetic field on isotropic MHD turbulence has been widely discussed in the literature (see e.g. [44-51]): it renders MHD turbulence anisotropic and slows energy decay $[49,51,52]$. This slow down reflects the reduction of energy transfer along the mean magnetic field direction. Note that two-dimensional turbulence might be more representative of strong MHD turbulence, where the cascade of energy occurs preferentially in the direction perpendicular to the mean magnetic field $\boldsymbol{B}$, than isotropic MHD turbulence with no mean magnetic field or MHD turbulence with a mean magnetic field that is not strong enough to impose a full anisotropic dynamics [53]. Cho et al. [49] found that the total energy decay in balanced strong MHD turbulence (i.e. with zero cross-helicity) follows a power law $E(t) \propto t^{-\alpha}$ where $\alpha$ is very close to unity. Bigot al. [51] predicted a slowing down of the energy decay due to anisotropy in the limit of strong mean magnetic field, with distinct powerlaws for energy decay of shear-Alfvén waves $\left(\sim t^{-2 / 3}\right)$ and pseudo-Alfvén waves $\left(\sim t^{-1}\right)$.

Figure 2 shows the time evolution of the total energy $E(t)$ for two values of the uniform magnetic field, $\mathrm{B}=0.2$ and 1.5 (runs 1 and 2 in table 2). In all our MHD simulations with or without rotation, the magnetic diffusivity is equal to kinematic viscosity $(\eta=\nu)$ so that the initial magnetic Reynolds number $\operatorname{Rem}=u_{l} l / \eta=\operatorname{Re}$ is about 170 (see table 1). In addition, the magnetic fluctuations are initially zero and hence the turbulent magnetic energy $E^{(m)}$ is initially zero. As observed, the decay of total energy is close to $E(t) \sim t^{-1.3}$ for $B=0.2$ and to $E(t) \sim t^{-1}$ for $B=1.5$. Therefore, the case with $B=0.2$ can be compared with a previous study devoted to pure isotropic turbulence since the mean field is not strong enough to impose a fully anisotropic dynamics [53]. According to the study by Banerjee and Jedamzik [42], the total energy in isotropic MHD turbulence decays as $t^{-1.3}$. In contrast, for the case with $B=1.5$, the total energy decay rate is close to unity as in the case of balanced strong MHD turbulence [49]. 


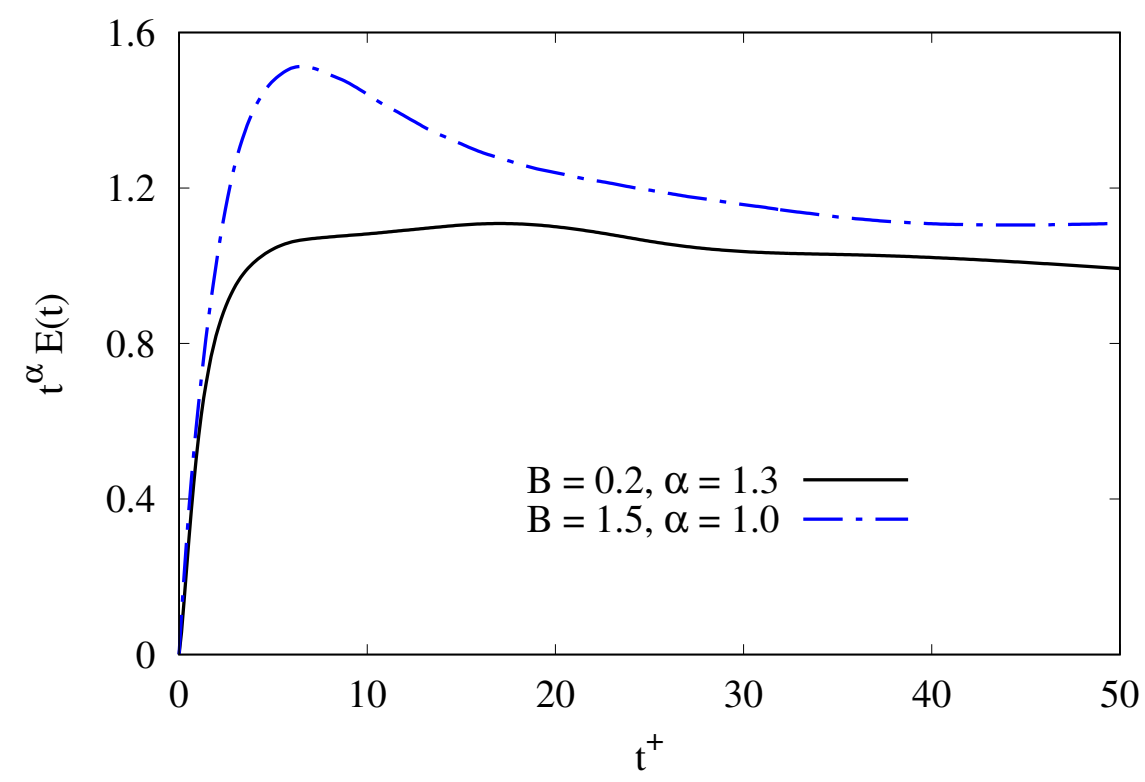

Figure 2. Time evolution of compensated total energy, $t^{\alpha} E(t)$ in nonrotating MHD turbulence with low $(B=0.2)$ or strong $(B=1.5)$ mean magnetic field.

\subsubsection{Rotating $M H D$ cases}

In the following, we analyze the effects of rotation on the MHD turbulence case with $B=0.2$ considering both configurations $(\boldsymbol{\Omega} \| \boldsymbol{B})$ and $(\boldsymbol{\Omega} \perp \boldsymbol{B})$. Three different values for the rotation rate are used, $\Omega=4.0,9.0$ and 16 (runs 3-8 in table 2).

In rotating hydrodynamic turbulence, a conventional measure of the strength of rotation is obtained by comparing the rotation time scale $\Omega^{-1}$ to the turbulence time scale $l / u_{l}$, yielding the turbulent Rossby number $R o=u_{l} /(2 l \Omega)$. In our simulations, the initial values of Rossby number are $\mathrm{Ro}=0.16,0.07$ and 0.04 (see also table 2). Typical large-scale planetary flows are characterized by Ro $\sim 0.1[54]$ and the liquid metals in the Earth's outer core are strongly affected by rotation with Ro $\sim 10^{-6}$ [55]. For a giant planet like Jupiter, it is believed that the Rossby number may even be smaller [56], whereas for the solar convective region (where the magnetic field is believed to be enhanced) Ro $\sim 1$ [5]. Another important parameter for rotating turbulence is the microscale Rossby number [20] $\operatorname{Ro}_{\omega}=\sqrt{\varepsilon^{(\kappa)} / \nu} /(2 \Omega)$ which is interpreted as the ratio of the convective to the Coriolis acceleration at the Taylor scale, and ought to be larger than one for scrambling effects of inertial waves not to completely damp nonlinear transfers [20]. The initial values of $R_{0}$ and $R_{\omega}$ are provided in table 2 .

In rotating MHD flows, the Elsasser number $\Lambda=B^{2} /(2 \Omega \eta)$ characterizes the relative strengths of the Lorentz and Coriolis forces and is commonly employed as a measure of the strong field dynamo, which is synonymous with the magnetostrophic balance (i.e., the pressure gradient, the Coriolis force and the Lorentz force are balanced). It is believed that the Elsasser number of the Earth's core is about unity [56]. For runs $(3,4),(5,6)$ and $(7,8)$ the respective values of the Elsasser number are $\Lambda=0.5$, 0.9 and 2 (see table 2). In homogeneous MHD turbulence, the Elsasser number cannot explicitly characterize the turbulence dynamics since it depends only on $\Omega, B$ and $\eta$ which are constants, and not on velocity and length scales that are required for assessing dynamical balances (see also the study of the convection-driven multi-scale dynamo model by Calkins et al. [57]).

Figure 3 shows the time evolution of total energy for the rotating MHD cases (runs 


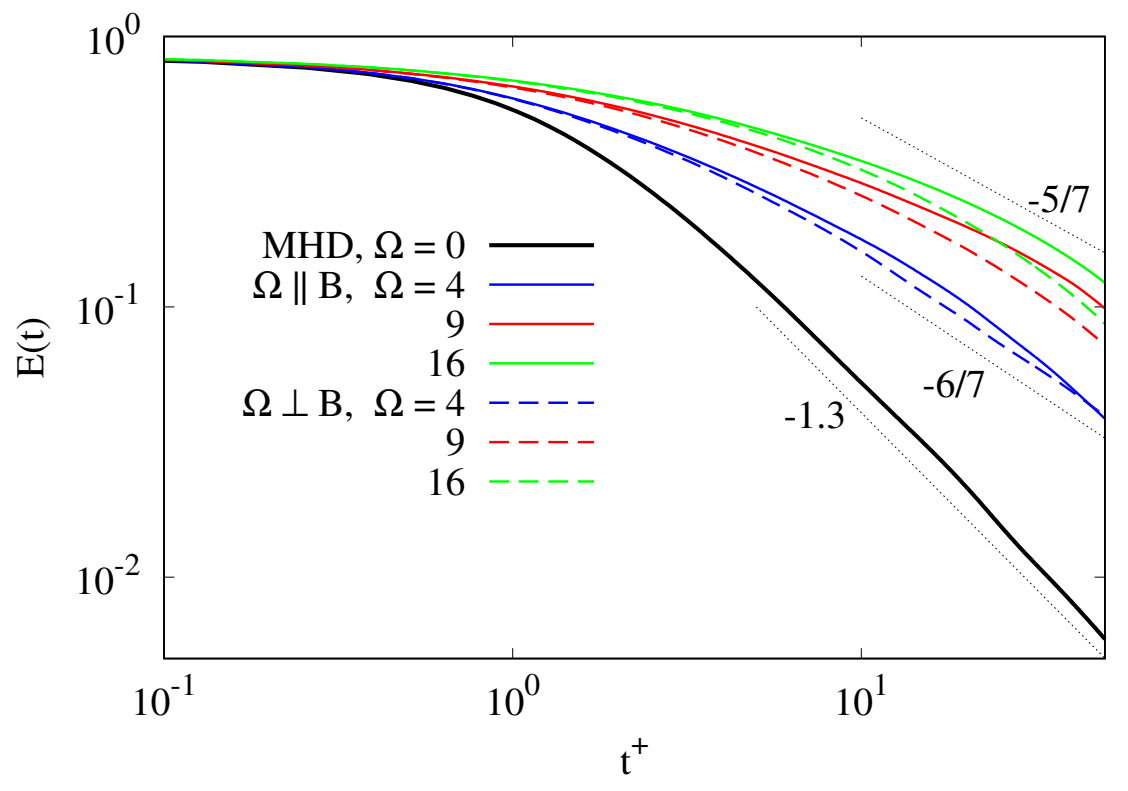

Figure 3. Time development of total (kinetic + magnetic) energy for the non-rotating (run 1 ) and rotating MHD cases for which the rotation vector is either parallel $(\boldsymbol{\Omega} \| \boldsymbol{B})$ or perpendicular $(\boldsymbol{\Omega} \perp \boldsymbol{B})$ (runs 3-8). The initial state corresponds to a homogeneous isotropic hydrodynamic turbulence with $k^{4}$ infrared energy spectrum.

3 to 8). DNS results for the non-rotating MHD case with $B=0.2$ (run 1) are also reported. The energy decrease is clearly slower for the rotating MHD cases than for the non-rotating case. The reduction of kinetic energy decay by rotation is well known in the non-magnetized case. For instance, the time decay exponent in homogeneous isotropic hydrodynamic turbulence is $-10 / 7$ for $k^{4}$ infrared energy spectrum and $-6 / 5$ for $k^{2}$ [35], and is almost exactly halved in the presence of rotation, whence $-5 / 7$ for $k^{4}$, from wave-turbulence theory [12], experiment [58,59] and DNS [60,61]. Our simulations show that the $-5 / 7$ exponent is recovered at large times for the highest rotation rate $(B=0.2, \Omega=9,16$, runs $5-8)$, while $-6 / 7$ is found at lower rotation rate $(B=0.2, \Omega=$ 4 , runs 3 and 4). Accordingly, we conclude that the decay exponent of total energy in rotating MHD turbulence with a mean magnetic field that is not strong enough follows these classical predictions for kinetic energy decay in rotating hydrodynamic turbulence. Moreover, the time development of total energy is not noticeably sensitive to the angle between the rotation vector and the mean magnetic field although the generation of magnetic fluctuations in the perpendicular case is more important than in the parallel one, as will be shown later.

For the same parametric cases as figure 3, figure 4 shows the time evolution of total dissipation rate $\varepsilon=\varepsilon^{(\kappa)}+\varepsilon^{(m)}$. During the initial phase $t^{+}<4, \varepsilon$ decreases as the rotation rate increases, whereas for $t^{+}>4$ the decay of $\varepsilon$ slows down as the rotation rate increases. At large times, the decay of $\varepsilon$ tends to the powerlaw $\varepsilon \sim t^{\alpha-1}$ where $(\alpha-1)=-2.3$ for run $1,(\alpha-1)=-13 / 7$ for runs 3 and 4 , and $(\alpha-1)=-12 / 7$ for runs 5-8, in agreement with the total energy equation, $\partial_{t} E=-\varepsilon$ which yields $\varepsilon \sim t^{\alpha-1}$ if $E \sim t^{\alpha}$.

The ratio $\mathrm{N}=\Lambda / \mathrm{Ro}=B^{2} l / \eta u_{l}$ is the interaction parameter or Stuart's number, and compares the diffusion time of the kinetic energy due to the Lorentz force to the eddy turnover time. In our simulations, $\mathrm{N}$ is observed to start at a moderate value $\mathrm{N} \approx 12$ for runs 1, 3-8 and to increase with time to about 170 at the final computational time (see inset of figure 4). 


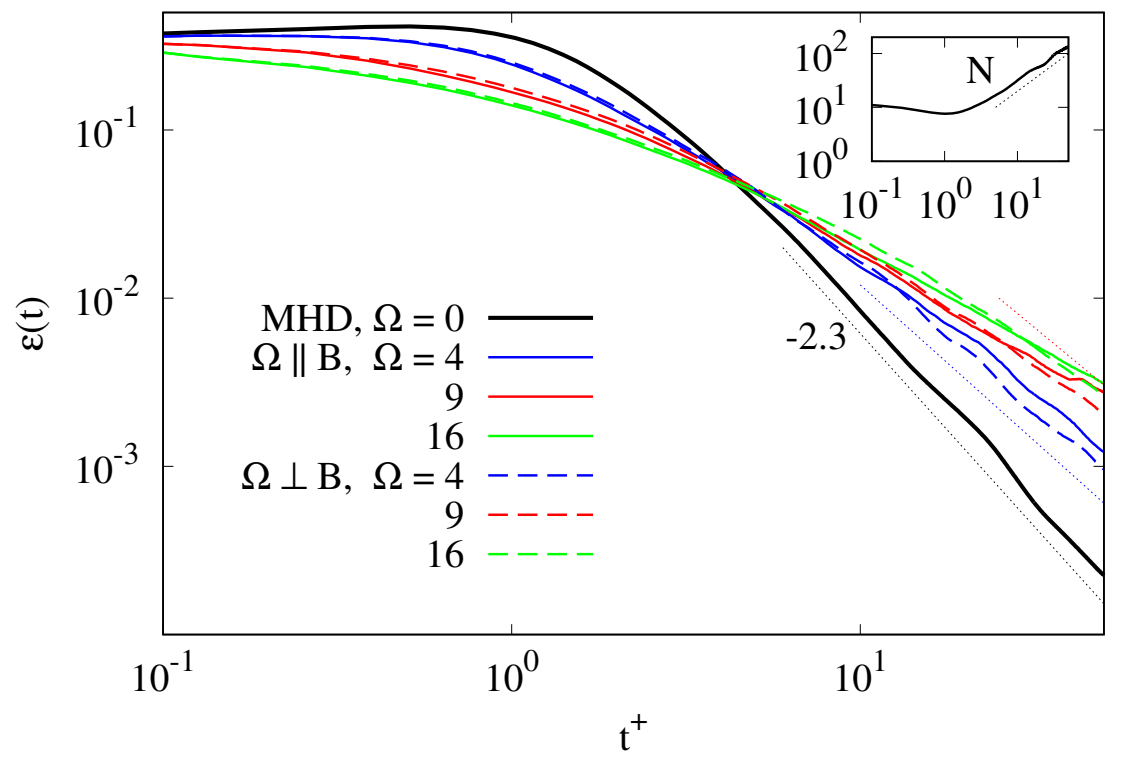

Figure 4. Time development of total (kinetic + magnetic) dissipation rate for the non-rotating (run 1 ) and rotating MHD cases for which the rotation vector is either parallel $(\boldsymbol{\Omega} \| \boldsymbol{B}$ ) or perpendicular $(\boldsymbol{\Omega} \perp \boldsymbol{B})$ (runs $3-8)$. From bottom to top, the straight lines indicate the power law decay $t^{-2.3}, t^{-13 / 7}$ and $t^{-12 / 7}$, respectively. The inset shows the time development of the interaction parameter $\mathrm{N}$ for the non-rotating MHD case, $\mathrm{N} \propto t$ for large times.

\subsubsection{Energy ratios}

Figures $5(\mathrm{a}, \mathrm{b})$ show the short-time development $\left(t^{+} \leq 5\right)$ of the Alfvén energy ratio $\Gamma(t)=E^{(\kappa)}(t) / E^{(m)}(t)$ for runs 1 and 3-6: in the non-rotating MHD case (run 1), there is a quasi-equipartition between kinetic and magnetic energies $(\Gamma \sim 1$, as in e.g. [7,53]). During the initial phase $t^{+}=u_{l 0} t / l_{0} \sim 4$, the transfer $\mathcal{F}^{(\kappa m)}$ falls rapidly, transferring nearly half its content to the magnetic energy $E^{(m)}(t)$, as also shown by figures $6(\mathrm{a}, \mathrm{b})$ displaying the time development of kinetic and magnetic energies normalized by total energy. After this initial phase $\left(t^{+}>4\right)$, the energy exchanges between kinetic and magnetic fluctuations, due to Alfvén waves, produce oscillations. The period of oscillations is $T^{+} \sim 20$ or equivalently $\tau_{A}=l_{A} / B \sim 16$ where $\tau_{A}$ is the Alfvén wave period and $l_{A} \sim \pi$ is a characteristic length scale and corresponds to
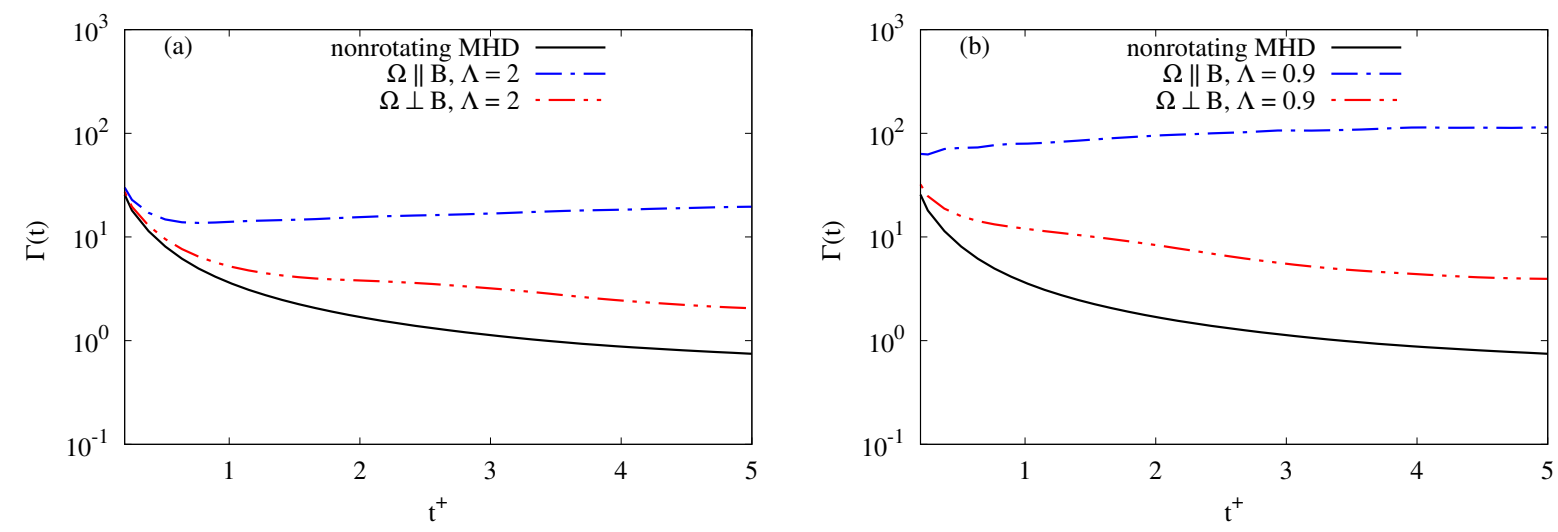

Figure 5. Time development of the energy Alfvèn ratio $\Gamma(t)=E^{(\kappa)} / E^{(m)}$ in nonrotating MHD case (run $1)$, parallel case $(\boldsymbol{\Omega} \| \boldsymbol{B}$, runs 3 and 5$)$ and perpendicular case $(\boldsymbol{\Omega} \perp \boldsymbol{B}$, runs 4 and 6$)$. 

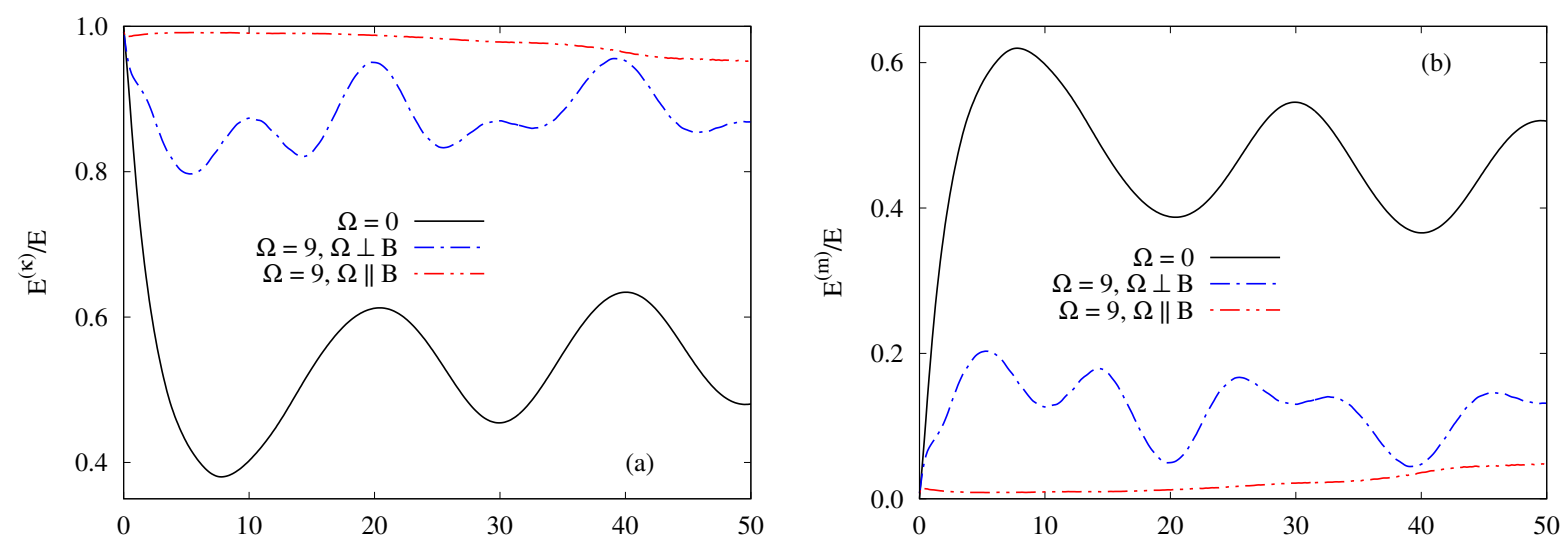

Figure 6. Time development of kinetic energy $E^{(\kappa)}$ and magnetic energy $E^{(m)}$ normalized by total energy $E$ in the non-rotating (run 1) and rotating MHD cases (runs 5 and 6).

half the box size. Note that in strongly magnetized flows, $l_{A}$ rather corresponds to a characteristic parallel length scale, where $\boldsymbol{B}$ defines the parallel direction [53].

Rotation causes a reduction of total energy as discussed previously and drastically reduces the energy exchanges between kinetic and magnetic components. Indeed, the quasi-equipartition between kinetic and magnetic energies is broken by inertial waves in both the parallel $(\boldsymbol{\Omega} \| \boldsymbol{B})$ and the perpendicular $(\boldsymbol{\Omega} \perp \boldsymbol{B})$ cases and the generation of magnetic fluctuations is substantially reduced especially in parallel case. For example, at $t^{+}=5$ and $\Lambda=0.9$ (runs 5 and 6 ), Alfvén energy ratio $\Gamma$ in the perpendicular case is 20 times larger than the one in the parallel case (see figure $5 \mathrm{~b}$ ).

To explain the reduction of the energy exchanges by rotation, theoretical insight can be gained by examining the dispersion relation of magneto-Coriolis waves described by equation (1) and reported here for the sake of clarity, as

$$
\omega_{f, s}=\Omega \frac{k_{\|}^{\Omega}}{k}\left(\sqrt{1+\left(\frac{k_{\|}^{B}}{k_{\|}^{\Omega}} \mathcal{L}_{k}\right)^{2}} \pm 1\right)
$$

where

$$
k_{\|}^{\Omega}=|\boldsymbol{k} \cdot \boldsymbol{\Omega}| / \Omega, \quad k_{\|}^{B}=|\boldsymbol{k} \cdot \boldsymbol{B}| / B,
$$

and reminding that $\mathcal{L}_{k}=B k / \Omega$ is a local Lehnert number. In the parallel case $k_{\|}^{B}=$ $k_{\|}^{\Omega}=\left|k_{3}\right|$, while in the perpendicular case $k_{\|}^{B}=\left|k_{1}\right|$ and $k_{\|}^{\Omega}=\left|k_{3}\right|$. At large scales $\left(\mathcal{L}_{k} \ll 1\right)$, where a great part of energy resides, and when $k_{\|}^{B} \neq 0$ and $k_{\|}^{\Omega} \neq 0$, inertial wave dynamics is dominant $\left(\omega_{f, s} \sim 2 \Omega k_{\|}^{\Omega} / k\right)$ causing a reduction of energy exchange between kinetic and magnetic components in both parallel and perpendicular cases. In addition, for the parallel case, the frequency of magneto-Coriolis waves vanish value $\left(\omega_{f, s}=0\right)$ at $k_{\|}^{\Omega}\left(=k_{\|}^{B}\right)=0$, the energy exchange due to the mean magnetic field also vanishes $\left(f^{(\kappa m)}=0\right)$, and the nonlinear transfer is strongly reduced (as in rotating hydrodynamic turbulence [12]). In counterpart, for the perpendicular case, Alfvén wave dynamics is dominant at $k_{\|}^{\Omega}=0$ since on this subset the frequency of magneto-Coriolis waves is reduced to the frequency of Alfvén waves $\omega_{f, s}=\omega_{A}=B k_{\|}^{B}$. The contribution coming from this mode in the generation of magnetic energy in the perpendicular case makes the magnetic energy relatively more important in this case than in the parallel 


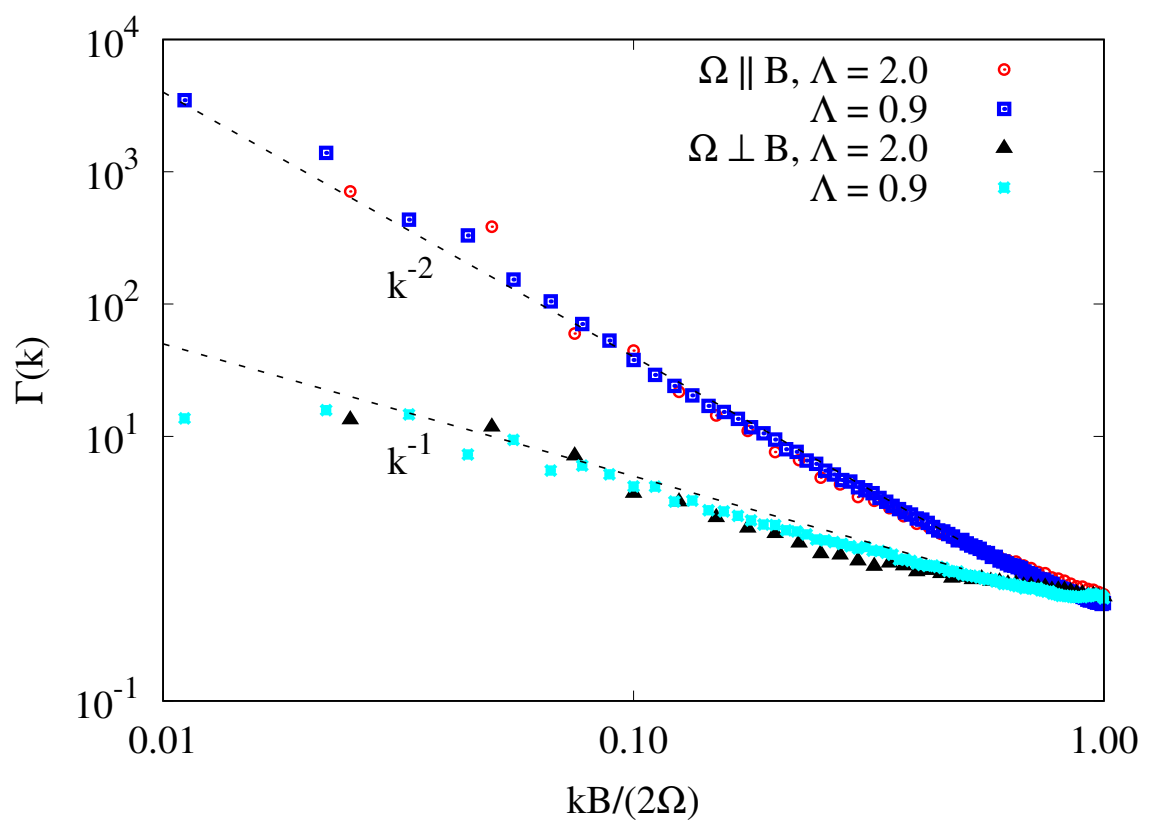

Figure 7. Local Alfvén energy ratio, $\Gamma(k, t)=S^{(\kappa)}(k, t) / S^{(m)}(k, t)$, versus $B k /(2 \Omega)$ at $t^{+}=2$ in parallel case $(\boldsymbol{\Omega} \| \boldsymbol{B}$, runs 3 and 5$)$ and perpendicular case $(\boldsymbol{\Omega} \perp \boldsymbol{b}$, runs 4 and 6$)$ Linear theory solution [8] indicates that $\Gamma(k, t)$ behaves as $k^{-2}$ in the parallel case and as $k^{-1}$ in the perpendicular case.

case. The competition between Alfvén and inertial waves in the perpendicular case is reflected by the behavior of the kinetic (or magnetic) energy where the oscillations due to Alfvén waves are modulated by the inertial waves, leading to a period of oscillations about half the one observed in the absence of rotation $(\sim \pi /(2 B))$.

Note that linear theory solution by Salhi et al. [8] predicts a breaking of the equipartition between kinetic and magnetic energies in rotating MHD cases and indicates that the reduction of magnetic energy is more important in parallel case $(\boldsymbol{\Omega} \| \boldsymbol{B})$ than in perpendicular one $(\boldsymbol{\Omega} \perp \boldsymbol{B})$. Indeed, it is found that, at $k B /(2 \Omega)<1$, the local Alfvén ratio, $\Gamma(k, t)=S^{(\kappa)}(k, t) / S^{(m)}(k, t)$, behaves as $k^{-2}$ in the parallel case and as $k^{-1}$ in the perpendicular case. For $k B /(2 \Omega)>1$, there is an equipartition between kinetic and magnetic energies. Here, $S^{(\kappa)}(k, t)$ (respectively, $S^{(m)}(k, t)$ ) is the radial spectrum of kinetic (magnetic) energy (see equation (15)). For times that are not very large $\left(t^{+} \lesssim 5\right)$, DNS results agree with linear theory predictions as shown by figure 7 .

\subsection{Spectra for kinetic and magnetic energies}

\subsubsection{Radial spectra}

In the section, we analyze radial spectra of kinetic and magnetic energies at different times. They provide information about the distribution of energy at different scales. The kinetic energy radial spectrum is integrated as

$$
S^{(\kappa)}(k, t)=k^{2} \int_{0}^{\pi} \int_{0}^{2 \pi} e^{(\kappa)}(\boldsymbol{k}, t) \sin \theta d \varphi d \theta
$$

where $(k, \theta, \varphi)$ is a spherical coordinates system for the wave vector $\boldsymbol{k}$. Similar definitions hold for other radial spectra.

Because the mean magnetic field $(B=0.2)$ is not strong enough to impose a full 

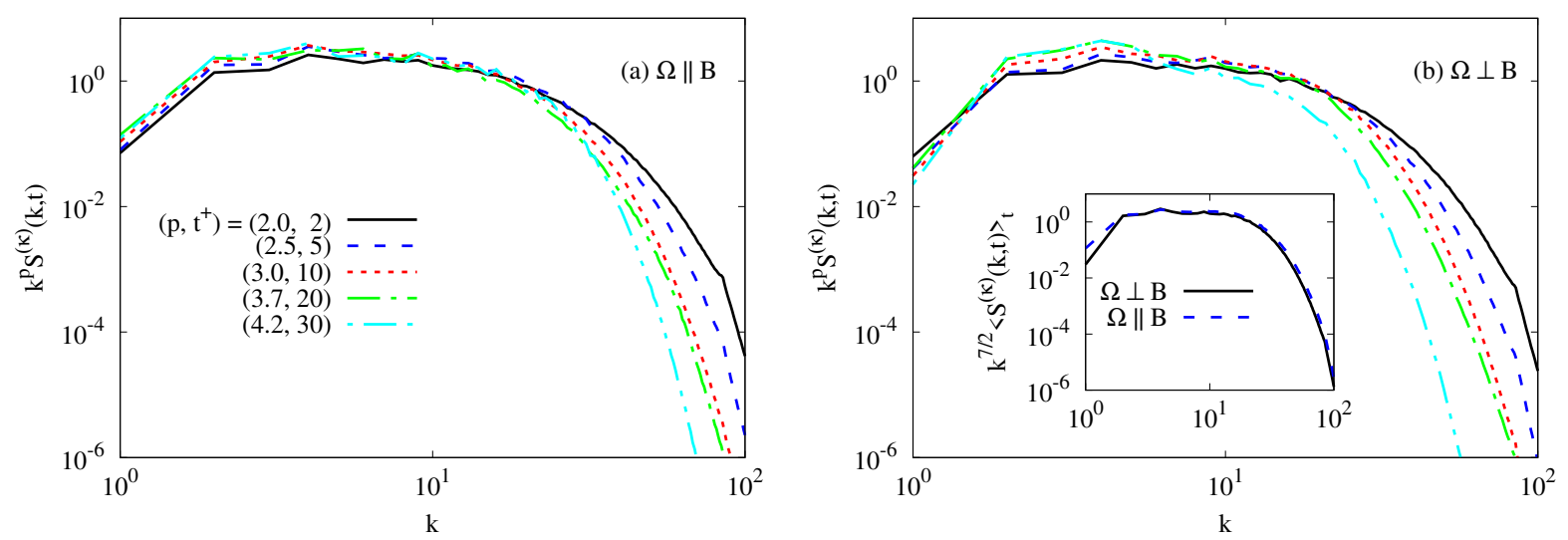

Figure 8. Compensated radial spectrum of kinetic energy, $k^{p} S^{(\kappa)}(k, t)$, at different times for (a) parallel case $\left(\boldsymbol{\Omega} \| \boldsymbol{B}\right.$, run 5) and (b) perpendicular case $\left(\boldsymbol{\Omega} \perp \boldsymbol{B}\right.$, run 6). The index $p$ increases as $t^{+}$increases, or equivalently, as the interaction parameter $\mathrm{N}$ increases. The inset shows that $\left\langle S^{(\kappa)}(k, t)\right\rangle_{t}$ behaves as $\sim k^{-7 / 2}$ at $2<k<20$ for both cases.

anisotropic dynamics, as already indicated, the energy spectrum $S(k, t)$ of total energy in non-rotating MHD (run 1) remains close to $\sim k^{-5 / 3}$.

Figures 6(a,b) shows plots of $k^{p} S^{(\kappa)}(k, t)$ in both the parallel $\boldsymbol{\Omega} \| \boldsymbol{B}$ and the perpendicular $\boldsymbol{\Omega} \perp \boldsymbol{B}$ cases, respectively at $\Omega=9$ and $B=0.2$ (runs 5,6). Accordingly, the Elsasser number is about $\Lambda=0.9$. The kinetic energy spectra are compensated with the exponents $p=2,2.5,3,3.7$ and 4.2 for $t^{+}=2,5,10,20$ and 30 respectively. In both the two flow configurations, the decay in kinetic energy $E(t)$ results in a reduction of the spectrum at all scales, especially at small scales. It is also clear that most of the energy is concentrated in the large scales. The spectrum $S^{(\kappa)}(k, t)$ behaves as $\sim k^{-p}$ for a decade of wavenumbers $(2<k<20)$ where the index $p$ increases as $t^{+}$increases, or equivalently, when the interaction parameter $\mathrm{N}=\left(B^{2} /(\pi \eta)\right)(E / \varepsilon)$ increases (see the inset of figure 4 that shows the increase of $\mathrm{N}$ with time). We note that the averaged spectrum $\left\langle S^{(\kappa)}(k, t)\right\rangle_{t}$ over the time window $t^{+} \in[5,50]$ shown by the inset of figure 7 (b) is closer to $\sim k^{-7 / 2}$ for $2<k<20$ in both flow configurations $\boldsymbol{\Omega} \| \boldsymbol{B}$ and $\boldsymbol{\Omega} \perp \boldsymbol{B}$.

The steepening of the energy spectrum (compared to Kolmogorov's spectrum) as the interaction parameter $\mathrm{N}$ increases has been observed in previous forced quasistatic (QS) MHD turbulence simulations $[2,62,63]$. Some authors attributed this steepening to the two-dimensionalization of QS MHD turbulence [64,65], while Verma and Reddy [66] indicated that it arises because of the decrease of the energy flux $\Pi(k)=\int_{0}^{k} \int_{0}^{\pi} \int_{0}^{2 \pi} k^{2} T(\boldsymbol{k}) \sin \theta d \varphi d \theta d k$ with $k$ due to the magnetic (Joule) dissipation. It can be recalled that the Alfvén waves are not only damped by the anisotropic Ohmic dissipation term, but completely vanish in the QSMHD case, so that the dynamics, with obvious two-dimensionalization, is generally very different from the dynamics of Alfvénic MHD (see also Favier et al. [67]).

As for figure 6(a,b), figure 7(a,b) show the magnetic energy spectrum $S^{(m)}(k, t)$. At large scales, $S^{(m)}(k, t)$ in perpendicular case is about $10^{2}$ times $S^{(m)}(k, t)$ in the parallel case. This is due in part to the fact that there are more exchanges between kinetic and magnetic fluctuations due to $\boldsymbol{B}$ in the perpendicular case than in the parallel one, as shown by figure 10 displaying the radial spectrum $F^{(\kappa m)}(k)$. Recall that $F^{(\kappa m)}(k)$ characterizes the energy exchanges between kinetic and magnetic components due the mean magnetic field. At small scales, $S^{(m)}(k, t)$ is approximately the same for both cases and $F^{(\kappa m)}(k, t)$ approaches zero. The magnetic energy time increase at low wavenumbers for the parallel case could indicate an inverse cascade of energy. 

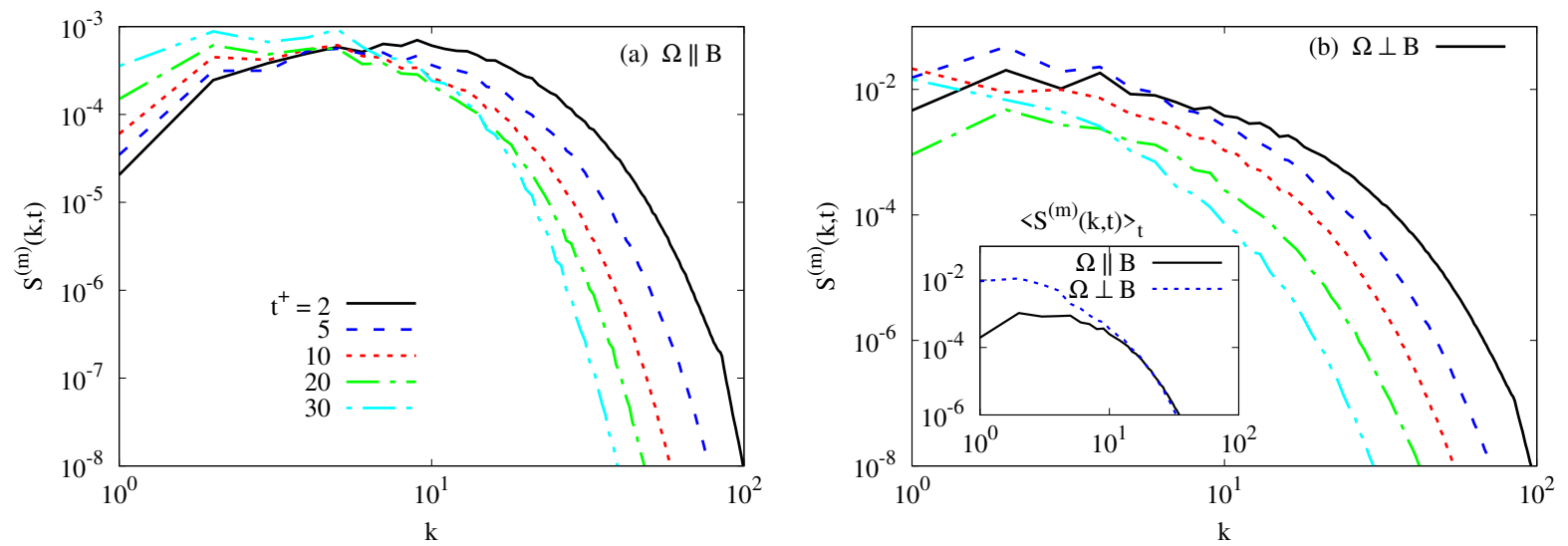

Figure 9. Radial spectrum of magnetic energy at different times for (a) parallel case ( $\boldsymbol{\Omega} \| \boldsymbol{B}$, run 5 ) and (b) perpendicular case $\left(\boldsymbol{\Omega} \perp \boldsymbol{B}\right.$, run 6). The inset shows the averaged over the time window $t^{+} \in[5,50]$ of the magnetic energy $\left\langle S^{(m)}(k, t)\right\rangle_{t}$ in both cases. It clealy appears that, at large scales, the generation of magnetic fluctuations is significantly more important in the perpendicular case than in the parallel one.

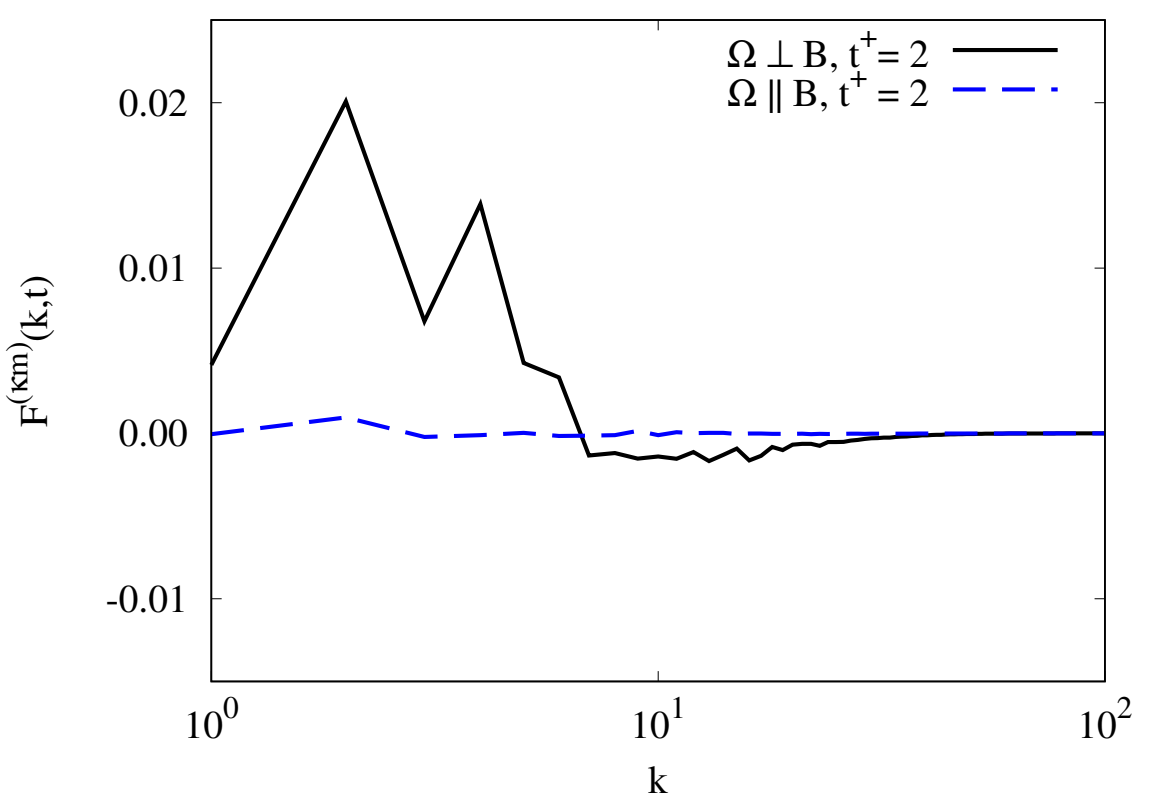

Figure 10. Radial spectrum $F^{(\kappa m)}(k, t)$ of energy exchanges due to $\boldsymbol{B}$ in parallel case $(\boldsymbol{\Omega} \| \boldsymbol{B}$, run 5$)$ and perpendicular case $(\boldsymbol{\Omega} \perp \boldsymbol{B}$, run 6$)$. It appears that the energy exchange due to $\boldsymbol{B}$ principally occurs at large scales and is significantly more important in perpendicular case than in parallel one. 

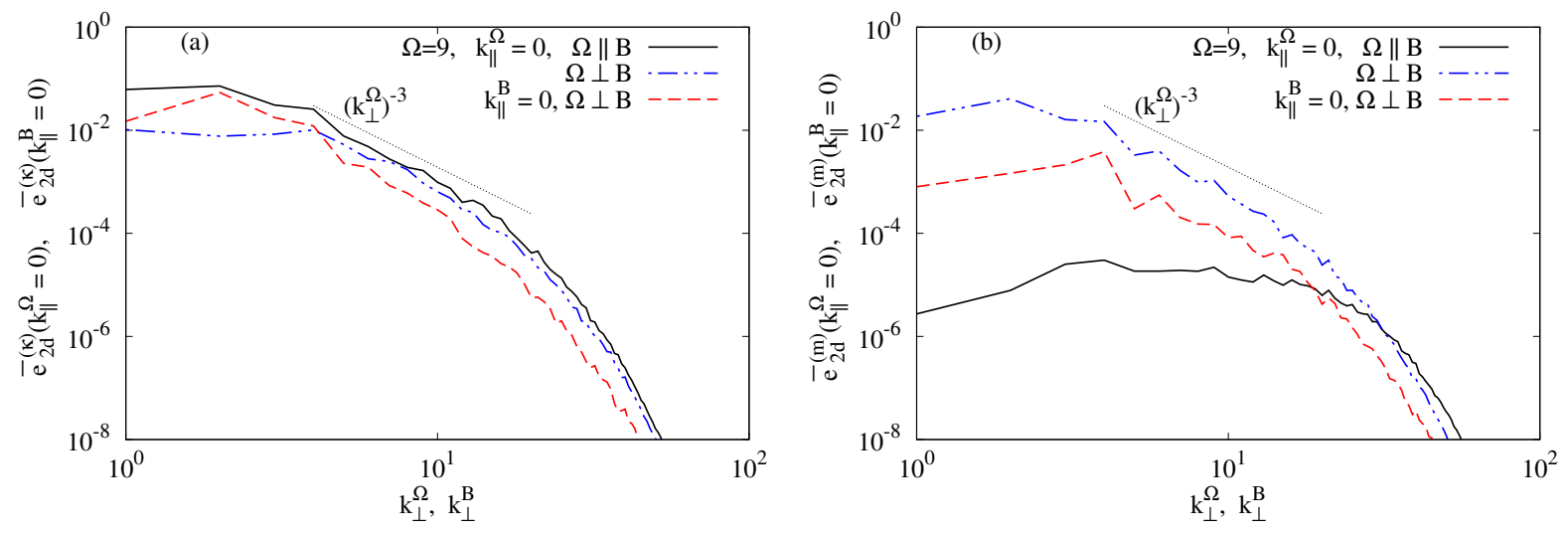

Figure 11. 2D energy spectra at $k_{\|}^{\Omega}=0$ or $k_{\|}^{B}=0$ for $t^{+}=5$ in parallel $(\boldsymbol{\Omega} \| \boldsymbol{B})$ and perpendicular $(\boldsymbol{\Omega} \perp \boldsymbol{B})$ cases (runs 5 and 6). (a) kinetic energy; (b) magnetic energy.

\subsubsection{2-D energy spectra at $k_{\|}^{\Omega}=0$}

The difference between parallel and perpendicular cases concerning the generation of magnetic fluctuations is more apparent when considering the 2D kinetic and magnetic energies in the plane $k_{\|}^{\Omega}=0$,

$$
\bar{e}_{2 d}^{(\kappa)}\left(k_{\perp}^{\Omega}, k_{\|}^{\Omega}=0\right)=\int_{0}^{2 \pi} e(\boldsymbol{k}, t) \delta\left(\boldsymbol{k}-\boldsymbol{k}_{\perp}^{\Omega}\right) d \varphi
$$

where $\delta\left(\boldsymbol{k}-\boldsymbol{k}_{\perp}^{\Omega}\right)$ is the Dirac function.

In the parallel case and at $k_{\|}^{\Omega}=0$, both inertial and Alfvén waves vanish, as noted previously, and energy exchanges due to $\boldsymbol{B}$ are zero, so that, $f_{2 d}^{(\kappa m)}\left(t, k_{\|}^{\Omega}=\right.$ $0)=0$. Therefore, the generation of magnetic fluctuations are only due to nonlinear interactions, but in the plane $k_{\|}^{\Omega}=0$, there is a slow down in the energy transfer rate because of interactions between waves (occurring for $k_{\|}^{\Omega} \neq 0$ ) and eddies (occurring for $k_{\|}^{\Omega}=0$ ) as for rotating hydrodynamic turbulence $[12,19,68]$. This explains the fact that the magnetic $2 \mathrm{D}$ energy at $k_{\|}^{\Omega}=0$ in the parallel case is very small compared to the one in the perpendicular case, as shown by figure 11(a),(b). Indeed, in the perpendicular case and at $k_{\|}^{\Omega}=0$, only inertial waves vanish and not Alfvén waves (see section 3.2.3), and hence energy exchanges are mainly due to Alfvén waves. Figure 11 also shows the two-dimensional magnetic energy at $k_{\|}^{B}=0$ in the perpendicular case. In that case, Alfvén waves vanish as well as the energy exchanges due to $\boldsymbol{B}$ (i.e. $\left.f^{(\kappa m)}\left(k_{\|}^{B}=0\right)=0\right)$, but inertial waves are present and the generation of magnetic fluctuations are rather due to nonlinear interactions. We observe that $\bar{e}_{2 d}^{(m)}\left(k_{\|}^{\Omega}=0\right)>$ $\bar{e}_{2 d}^{(m)}\left(k_{\|}^{B}=0\right)$ at almost all scales. As in several rotating hydrodynamic flows (rotating Rayleigh-Bénard convection, or large atmospheric scales), in the plane $k_{\|}^{\Omega}=0$ the kinetic energy spectrum scales as $\left(k_{\perp}^{\Omega}\right)^{-3}$ (see figure 11(a)). However, only in the perpendicular case does the magnetic energy exhibits such a scaling (see figure 11(b)). 


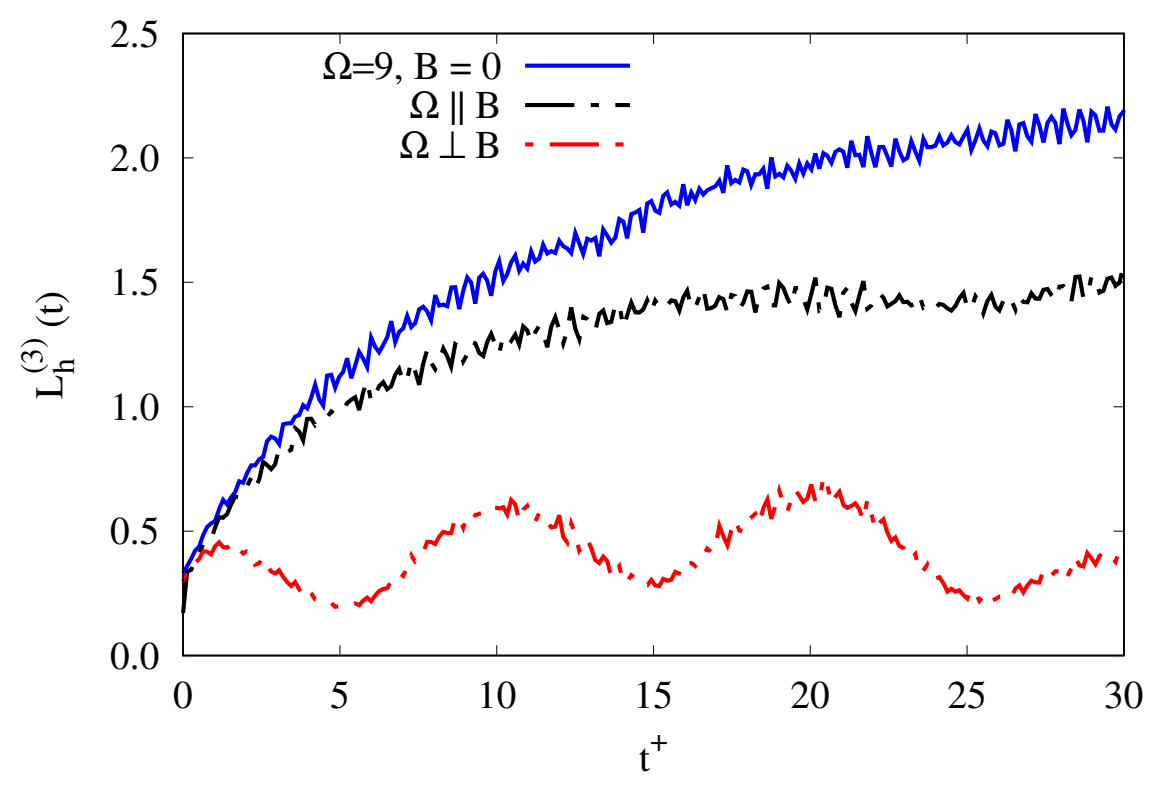

Figure 12. Time development of the integral length scale $L_{h}^{(3)}(t)=L_{11}^{(3)}+L_{22}^{(3)}$ in rotating hydrodynamic turbulence (run 9), parallel case $(\boldsymbol{\Omega} \| \boldsymbol{B}$, run 5) and perpendicular case $(\boldsymbol{\Omega} \perp \boldsymbol{B}$, run 6$)$.

\subsection{Anisotropy}

In rapidly rotating hydrodynamic turbulence, anisotropy appears in the form of structures elongated along the axis of rotation $[21,58]$. The quantitative statistical characterization of the elongation of structures can be done using the integral length scales (see equation (11)), or equivalently,

$$
L_{j j}^{(3)}=\frac{2 \pi}{\left\langle u_{j} u_{j}\right\rangle} \int_{\mathbb{R}^{2}}\left\langle\hat{u}_{j} \hat{u}_{j}^{*}\right\rangle_{k_{\|}^{\Omega}=0} d k_{1} d k_{2},
$$

considering only the integral length scale in the direction parallel to the rotation axis, since $L_{11}^{(3)}=L_{22}^{(3)}$ increase due to rotation [11]. Figure 12 shows the time evolution of $L_{h}^{(3)}=L_{11}^{(3)}+L_{22}^{(3)}$. In the parallel case, $L_{h}^{(3)}(t)$ increases but more slowly than its counterpart in a rotating hydrodynamic case. For these two flow cases, $L_{33}^{(3)}(t)$ decreases with time (not shown). In the perpendicular case, both $L_{h}^{(3)}(t)$ and $L_{33}^{(3)}(t)$ oscillate with time about their initial values $\left(L_{h}^{(3)}(0)=L_{33}^{(3)}(0)\right)$, with a period $\sim \pi /(2 B)$, as the oscillations of energy ratios in the perpendicular case (see section 3.2.3). We illustrate this integral length scales anisotropy with a visualization, in figure 13, of the iso-surfaces of the magnitude of the vorticity $|\boldsymbol{\omega}|$, where $\boldsymbol{\omega}=\nabla \times \boldsymbol{u}$, in the horizontal $(x, y)$ plane at $t^{+}=30$ and $\Omega=9$ (runs 5, 6 and 9 ). The elongated structures along the rotation axis observed in rotating hydrodynamic turbulence (top panel in figure 13) are affected by the background magnetic field: their intensity is reduced by rotation in the parallel case (midle panel) and they are distorted or inhibited in the perpendicular case (bottom panel). [2],

To quantify the anisotropy of kinetic and magnetic energies, one may use the ratios

$$
A_{\kappa, m}(t)=\frac{E_{11}^{(\kappa),(m)}(t)+E_{22}^{(\kappa),(m)}(t)}{2 E_{33}^{(\kappa),(m)}(t)}, \quad E_{j j}^{(\kappa)}=\left\langle u_{j} u_{j}\right\rangle, \quad E_{j j}^{(m)}=\left\langle b_{j} b_{j}\right\rangle
$$



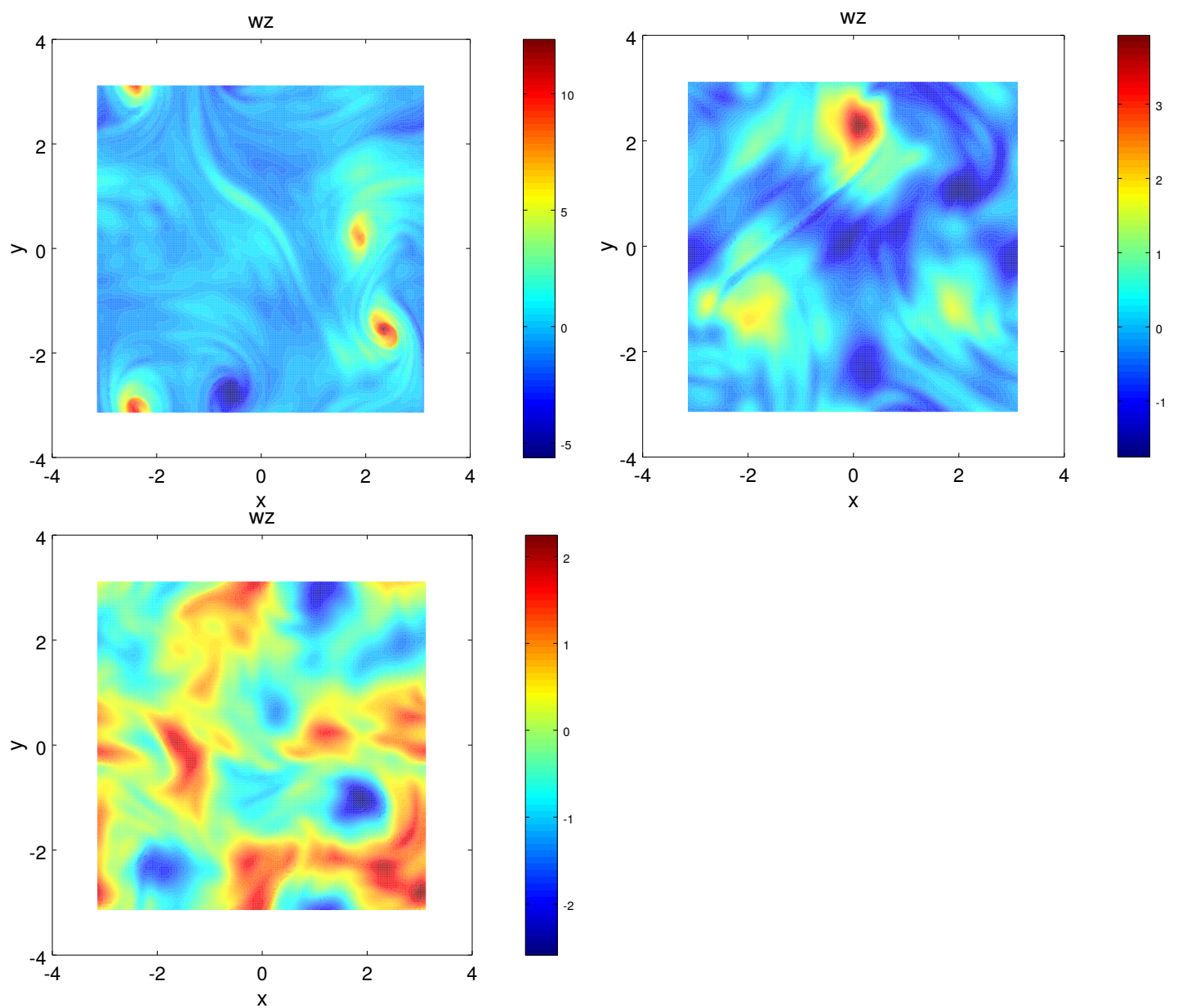

Figure 13. Horizontal cut of vertical vorticity $\omega_{z}$ in rotating hydrodynamic case (run 9)), parallel and perpendicular cases (runs 5 and 6 ). The elongated structures along the rotation axis observed in rotating hydrodynamic case (top pannel) are affected by the mean magnetic field: their intensities is weakened by rotation in the parallel case (middle panel) or they are distorted or inhibited in the perpendicular case (bottom panel). 

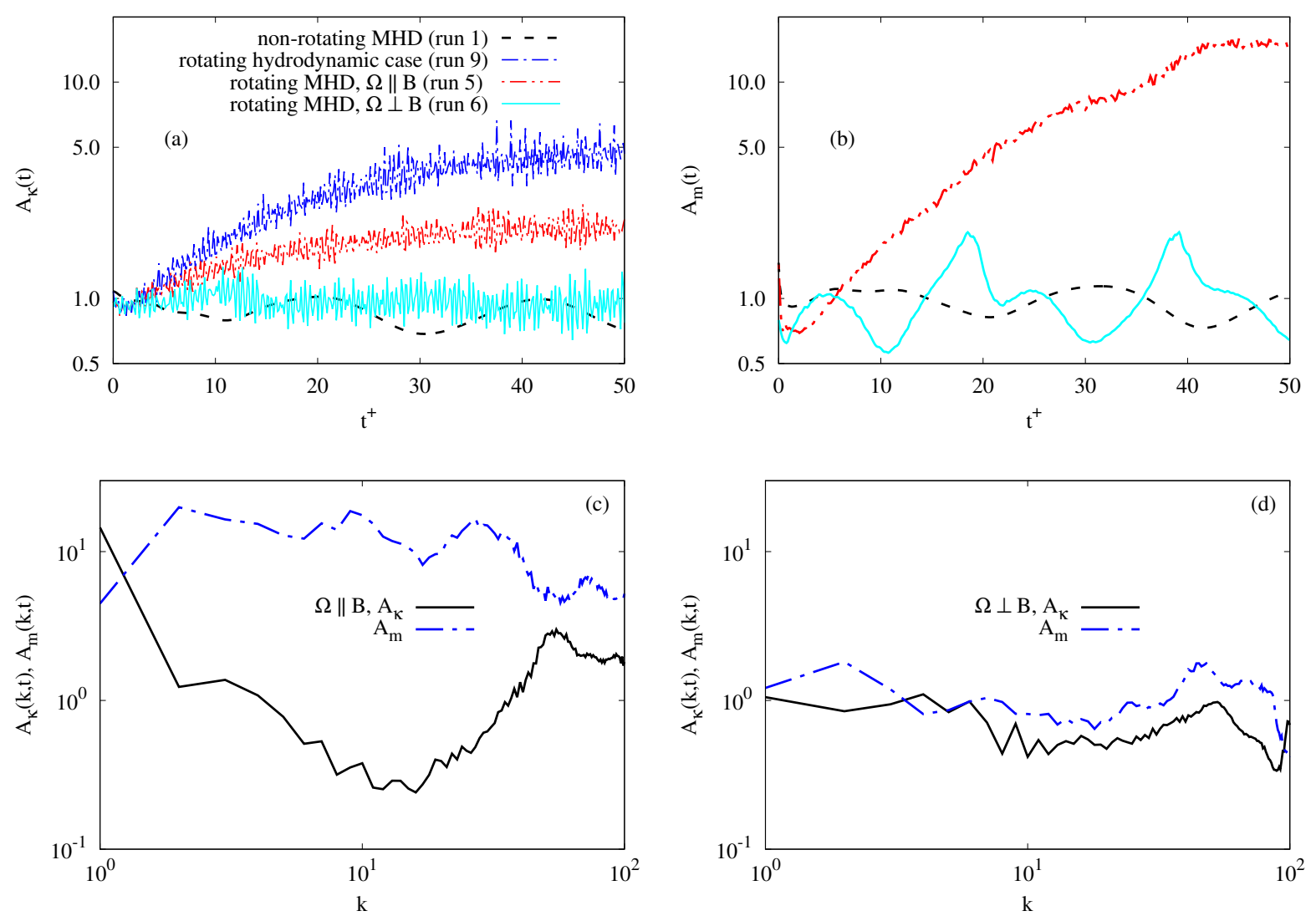

Figure 14. (a), (b) Time development of horizontal to vertical kinetic (respectively, magnetic) energies in rotating hydrodynamic case (run 9), non-rotating MHD case (run 1) and rotating MHD cases $(\boldsymbol{\Omega} \| \boldsymbol{B}$, run 5 and $\boldsymbol{\Omega} \perp \boldsymbol{B}$, run 6). (c), (d) plots of anisotropy spectrum of the velocity and magnetic fields in rotating MHD cases (runs 5 and 6 ) at large times $t^{+}=40$.

which are unity for isotropic MHD turbulence $\left(A_{\kappa}=A_{m}=1\right)$ but deviate from unity for anisotropic flows. Figures 14(a,b) show the time evolution of $A_{\kappa}(t)$ for both parallel and perpendicular cases (runs 5 and 6). DNS results for non-rotating MHD case (run 1 ) and rotating hydrodynamic case (run 9 ) are also reported for comparison.

For the parallel case $(\boldsymbol{\Omega} \| \boldsymbol{B}$, runs 5$), A_{\kappa}(t)$ increases towards the value 3 at large times, but remains less than $A_{\kappa}$ in the rotating hydrodynamic case (where $A_{\kappa}$ approaches 5 at large times, run 9 ). Scale by scale, we observe a strong decrease of the ratio

$\mathcal{A}_{\kappa, m}(k, t)=\frac{S_{11}^{(\kappa),(m)}+S_{22}^{(\kappa),(m)}}{2 S_{33}^{(\kappa),(m)}}, \quad S_{j j}^{(\kappa),(m)}(k, t)=k^{2} \int_{0}^{\pi} \int_{0}^{2 \pi}\left(\left\langle\hat{u}_{j} \hat{u}_{j}^{*}\right\rangle,\left\langle\hat{b}_{j} \hat{b}_{j}^{*}\right\rangle\right) \sin \theta d \varphi d \theta$.

Indeed, at $k<4$, the horizontal kinetic energy $\left(S_{11}^{(\kappa)}+S_{22}^{(\kappa)}\right)$ largely dominates the vertical one (see figure 14(c)). However, at $4<k<40$, it is the vertical kinetic energy that becomes dominant $\left(\mathcal{A}_{\kappa} \sim 0.2\right.$ at $\left.k=20\right)$. This strong decrease characterizes a two-dimensional and three-component $(2 \mathrm{D}-3 \mathrm{C})$ flow. The fact that the magnetic energy ratio $\mathcal{A}_{m}(k)$ is larger than 5 almost at all scales (figure $14(\mathrm{c})$ ) is not due to an excess of horizontal magnetic energy but rather due to weaker vertical magnetic energy at almost all scales.

For the perpendicular case ( $\boldsymbol{\Omega} \perp \boldsymbol{B}$, run 6$)$ both $A_{\kappa}(t)$ and $A_{m}(t)$ exhibit an oscillatory behavior around unity (see figures $14(\mathrm{a}),(\mathrm{b})$ ) with an amplitude greater than the one observed for the non-rotating MHD case. The behavior of $\mathcal{A}_{\kappa}(k)$ indicates 
that, at $k>6$, the vertical kinetic energy slightly dominates the horizontal one. The anisotropy in the perpendicular case is not as pronounced as in the parallel case.

\section{Concluding remarks}

The present study gives new insights into nonlinear dynamics for rotating MHD turbulence, that extends the linear, mainly analytical, study of magneto-Archimedes-Coriolis waves[8]. As in the previous study, we investigated the case where the mean magnetic field is orthogonal to the angular velocity of the rotating frame $(\boldsymbol{\Omega} \perp \boldsymbol{B})$, in addition to the more classical case where they are aligned $(\boldsymbol{\Omega} \| \boldsymbol{B})$.

Our DNS start from isotropic hydrodynamic turbulence with a Taylor microscale Reynolds number about $\mathrm{R}_{\lambda} \sim 70$. In the nonrotating MHD case with a mean magnetic field that is not strong enough $(B=0.2)$ to impose a full anisotropic dynamics, the total (kinetic + magnetic) energy decays with time as $t^{-1.3}$. In contrast, for a strong mean magnetic field $(B=1.5)$, the decay rate of total energy is close to unity, in agreement with previous DNS results in the case of balanced strong MHD turbulence[49].

In the present work, rotation effects on MHD turbulence are studied only for the case where $B=0.2$ and three rotation rates $\Omega=4,9$ and 16 , or equivalently, in terms of the Elsasser number, $\Lambda=2,0.9$ and 0.5 , respectively. It is found that, for both parallel and perpendicular MHD cases, the total energy decay remains very similar to the kinetic energy decay in rotating hydrodynamic turbulence.

Rotation drastically reduces the energy exchanges between kinetic and magnetic components especially in the parallel case $(\boldsymbol{\Omega} \| \boldsymbol{B})$. Consequently, the generation of magnetic fluctuations is more important in the parallel case than in the perpendicular case. The analysis of the dispersion relation of magneto-Coriolis waves (equation (13)) indicates that, at large scales, inertial waves dominate Alfvén waves when $k_{\|}^{\Omega} \neq 0$ for both parallel and perpendicular cases. At $k_{\|}^{\Omega}=0$, the dispersion frequency of magnetoCoriolis waves is zero in the parallel case, but it reduces to the dispersion frequency of Alfvén waves in the perpendicular cases. Therefore, in the latter case $(\boldsymbol{\Omega} \perp \boldsymbol{B})$, the competition between inertial waves and Alfvén waves induces modulated oscillations with period $\pi /(2 B)$. Note that, in nonrotating MHD case, the energy exchanges, due to Alfvén waves, produce oscillations with period $\tau_{A}=\pi / B \sim 16$ (figure $6(\mathrm{a}, \mathrm{b})$ ).

In both parallel and perpendicular cases, the radial spectrum of kinetic energy exhibits a power law decay, $S^{(\kappa)}(k, t) \propto k^{-p}$, at $2<k<20$ where the exponent $p$ increases with times, or equivalently, as the interaction parameter $\mathrm{N}$ increases (see figure $8(\mathrm{a}, \mathrm{b}))$. This is very similar to the quasi-static MHD case. The spectrum $\left\langle S^{(\kappa)}(k, t)\right\rangle_{t}$ averaged over the time window $t^{+} \in[5,50]$ is close to $\sim k^{-7 / 2}$ at $2<k<20$. The $2 \mathrm{D}$ kinetic energy spectrum at $k_{\|}^{\Omega}=0$ behaves like $\left(k_{\perp}^{\Omega}\right)^{-3}$ reflecting the fact that system rotation favors two-dimensionalization.

As for the generation of magnetic fluctuations, it is found that, in the parallel case $(\boldsymbol{\Omega} \| \boldsymbol{B})$, the radial spectrum $S^{(m)}(k, t)$ increases with time at large scales, whereas it decreases at small scales (large wavenumbers, figure $9(\mathrm{a})$ ). The increase of $S^{(m)}(k, t)$ would characterize an inverse cascade of magnetic energy at large scales. The magnetic energy level at large scales is very small compared to the one in the perpendicular case $(\boldsymbol{\Omega} \perp \boldsymbol{B})$.

Finally, the anisotropy generated in the parallel (aligned) case is globally larger than the one in the perpendicular case. The elongated structures occurring in rapidly non-magnetized rotating flows are distorted or inhibited in the case where $\boldsymbol{\Omega} \perp \boldsymbol{B}$, and their intensity is weakened in the case where $\boldsymbol{\Omega} \| \boldsymbol{B}$. 


\section{References}

[1] Zimmerman D S, Triana S A, Nataf H-C and Lathrop D. A turbulent, high magnetic Reynolds number experimental model of Earth's core. J Geophys. Res: Solid Earth 2014;119(6):4538-4557.

[2] Verma M K. Anisotropy in Quasi-Static Magnetohydrodynamic Turbulence. Rep. Prog. Phys. 2017;80(8):087001.

[3] Plunian F and Stepanov R. Cascades and dissipation ratio in rotating MHD turbulence at low magnetic Prandtl number. Phys. Rev. E 2010;82(4):046311.

[4] Finlay C C. Waves in the presence of magnetic fields, rotation, and convection, in Dynamos, Vol. LXXXVIII of Lecture Notes of the Les Houches Summer School, edited by P Cardin and L Cugliandolo (Elsevier, New York), Chap. 8. 2008;403-450.

[5] Galtier S. Weak turbulence theory for rotating magnetohydrodynamics and planetary dynamos. J. Fluid Mech. 2014;757:114-154.

[6] Braithwaite J and Spruit H C. Magnetic fields in non-convective regions of stars. R Soc. Open Sci. 2017;4(2):160271.

[7] Favier B, Godeferd F, and Cambon C. On the effect of rotation on magnetohydrodynamic turbulence at high magnetic Reynolds number. Geophys. Astrophys. Fluid Dyn. 2012;106(1):89-111.

[8] Salhi A, Baklouti F S, Godeferd F, Lehner T and Cambon C. Energy partition, scale by scale, in magnetic Archimedes Coriolis weak wave turbulence. Phys. Rev. E 2017;95(2):023112.

[9] Galtier S, Nazarenko S V, Newell A C and Pouquet A. A weak turbulence theory for incompressible magnetohydrodynamics. J. Plasma Phys. 2000;63(5):447-488.

[10] Galtier S. Weak inertial-wave turbulence theory. Phys. Rev. E 2003;68(1):015301.

[11] Cambon C, Rubinstein R and Godeferd F S. Advances in wave turbulence: rapidly rotating flows. New Journal of Physics 2004;6(1):73.

[12] Bellet F, Godeferd F S, Scott J F and Cambon C. Wave-turbulence in rapidly rotating flows. J. Fluid Mech. 2006;562:83-121.

[13] Nazarenko S. Wave Turbulence. Lecture Notes in Physics. Berlin Springer Verlag. 2011.

[14] Perez J C, Mason J, Boldyrev S and Cattaneo F. On the Energy Spectrum of Strong Magnetohydrodynamic Turbulence. Phys. Rev. X 2012;2(4):041005.

[15] Shebalin JV, Matthaeus WH and Montgomery D. Anisotropy in MHD turbulence due to a mean magnetic field. J. Plasma Phys. 1983;29(3):525-547.

[16] Alexakis A. Two-dimensional behavior of three-dimensional magnetohydrodynamic flow with a strong guiding field. Phys. Rev. E 2011;84(5):056330.

[17] Gallet B and Doering C R. Exact two-dimensionalization of low-magnetic-Reynoldsnumber flows subject to a strong magnetic field. J. Fluid. Mech.2015;773:154-177.

[18] Sundar S, Verma K M, Alexakis A and Chatterjee A G. Dynamic Anisotropy in MHD Turbulence induced by mean magnetic field. Phys. Plasmas 2017;24(2):022304.

[19] Godeferd F S and Moisy F. Structure and Dynamics of Rotating Turbulence: A Review of Recent Experimental and Numerical Results. Appl. Mech. Rev. 2015;67(3):030802.

[20] Cambon C, Mansour N N, and Godeferd F S. Energy Transfer in Rotating Turbulence. J. Fluid Mech. 1997;337:303-332.

[21] Biferale L, Bonaccorso F, Mazzitelli I M, van Hinsberg M A T, Lanotte A S, Musacchio S, Perlekar P, and Toschi F. Coherent Structures and Extreme Events in Rotating Multiphase Turbulent Flows. Phys. Rev. X 2016;4:041036.

[22] Shebalin J V. Ideal homogeneous magnetohydrodynamic turbulence in the presence of rotation and a mean magnetic field. J. Plasma Phys. 2006;72(4):507-524.

[23] Nornberg M D, Ji H, Schartman E, Roach A, and Goodman J. Observation of Magnetocoriolis Waves in a Liquid Metal Taylor- Couette Experiment. Phys. Rev. Lett. 2010;104(7):074501.

[24] Balbus S A and Hawley J F. A powerful local shear instability in weakly magnetized disks. I-Linear analysis. Astrophys. J 1991;376214-233.

[25] Salhi A, Lehner T, Godeferd F and Cambon C. Magnetized rotating stratified shear waves. Phys. Rev. E 2012;85(2):026301.

[26] Lehnert B. The decay of magnetoturbulence in the presence of a magnetic field and 
Coriolis force. Q Appl. Math. 1955;12(4):321-341.

[27] Moffatt H K. Field Generation in Electrically Conducting Fluids. Cambridge University Press, Cambridge/London/New York/Melbourne 1978;2:5-1.

[28] Kraichnan R H. Inertial-Range spectrum of hydomagnetic turbulence. Phys. Fluids 1965; 8(7):1385-1387.

[29] Biskamp D. Nonlinear Magnetohydrodynamics. CUP, 1993.

[30] Baerenzung J, Politano H, Ponty Y, Pouquet A. Spectral Modeling of Magnetohydrodynamic Turbulent Flows. Phys. Rev. E 2008;78(2):026310.

[31] Lesur G and Longaretti P-Y. Impact of dimensionless numbers on the efficiency of magnetorotational instability induced turbulent transport. Mon. Not. R Astron. Soc. 2007;378(4):1471-1480.

[32] Rogallo R S, NASA TM 81315, 1981 (unpublished).

[33] Salhi A, Jacobitz F G, Schneider K and Cambon C. Nonlinear dynamics and anisotropic structure of rotating sheared turbulence. Phys. Rev. E 2014;89(1):013020.

[34] Khlifi A, Salhi A,Nasraoui S, Godeferd F and Cambon C. Spectral energy scaling in precessing turbulence. Phys. Rev. E 2018;98(1):011102.

[35] Sagaut P and Cambon C. Homogeneous Turbulence Dynamics. Cambridge University Press, Cambridge 2008.

[36] Pouquet A. Turbulence, statistics and structures: an introduction. In Vth European School in Astrophysics, Lecture Notes in Physics. Plasma Astrophysics. Springer 1996;468:163-212.

[37] Galtier S, Politano H and Pouquet A. Self-similar energy decay in magnetohydrodynamic turbulence. Phys. Rev. Lett. 1997;79(15):2807-2810.

[38] Pouquet A, Rosenberg D, Stawarz JE and Marino R. Helicity dynamics, inverse and bi-directional cascades in fluid and MHD turbulence: A brief review. Earth and Space Science 2019; 6(2):351-369.

[39] Biskamp D. Magnetohydrodynamic Turbulence. Cambridge University Press (2003).

[40] Muller W C and Biskamp D. Scaling properties of three-dimensional magnetohydrodynamic turbulence. Phys. Rev. Lett. 2000:84(3):475.

[41] Companelli L. Scaling laws in magnetohydrodynamic turbulence. Phys. Rev. D 2004:70(8):083009.

[42] Banerjee R and Jedamzik K. Evolution of cosmic magnetic fields: from the very early universe, to recombination, to the present. Phys. Rev. D 2004;70(12):123003.

[43] Briard A and Gomez T. The decay of isotropic magnetohydrodynamics turbulence and the effects of cross-helicity. J. Plasma Phys. 2018;84(1):905840110.

[44] Montgomery D and Turner L. Anisotropic magnetohydrodynamic turbulence in a strong external magnetic field. Phys. Fluids 1981;24(5):825-831.

[45] Shebalin JV,Matthaeus WH and Montgomery D. Anisotropy in MHD turbulence due to a mean magnetic field. J. Plasma Phys. 1983;29(3):525-547.

[46] Hossain M, Gray P C, Pontius D H and Matthaeus W H. Phenomenology for the decay of energy containing eddies in homogeneous MHD turbulence. Phys. Fluids 1985;7(11):28862904.

[47] Matthaeus W H, Oughton S, Ghosh S and Hossain M. Scaling of Anisotropy in Hydromagnetic Turbulence. Phys. Rev. Lett. 1998;81(10):2056-2059.

[48] Galtier S, Nazarenko SV, Newell AC and Pouquet A. A weak turbulence theory for incompressible magnetohydrodynamics. J. Plasma Phys. 2000;63(5):447-488.

[49] Cho J, Lazarian A and Vishniac E T. Simulations of magnetohydrodynamic turbulence in a strongly magnetized medium. Astrophys. J 2002:564(1):291-301.

[50] Verma M K. Statistical theory of magnetohydrodynamic turbulence: recent results. Phys. Rep. 2004;401(5-6):229-380.

[51] Bigot B, Galtier S and Politano H. Energy Decay Laws in Strongly Anisotropic Magnetohydrodynamic Turbulence. Phys. Rev. Lett. 2008;100(7):074502.

[52] Oughton S, Priest E R, Matthaeus W H. The influence of a mean magnetic field on threedimensional magnetohydrodynamic turbulence. J. Fluid Mech. 1994;280:95-117.

[53] Bigot B,Galtier S and Politano H. Development of anisotropy in incompressible magnetohydrodynamic turbulence. Phys. Rev. E 2008;78(6):066301.

[54] Shirley J H and Fairbridge R W. Encyclopedia of Planetary Sciences. Springer-Verlag, 
Berlin Heidelberg 1997;1020.

[55] Roberts P H and King E M. On the genesis of the Earth's magnetism. Reports Prog. Physics 2013;76(9):096801.

[56] Jones C. Planetary magnetic fields and fluid dynamos. Annu. Rev. Fluid Mech. 2011;43:583-614.

[57] Calkins M A, Julien K, Tobias S M and Aurnou J M. A multiscale dynamo model driven by quasi-geostrophic convection. J. Fluid Mech. 2015;780:143-166.

[58] Jacquin L, Leuchter O, Cambon C and Mathieu J. Homogeneous turbulence in the presence of rotation. J. Fluid Mech. 1990;220:1-52.

[59] C Morize, F Moisy and M Rabaud, Decaying grid-generated turbulence in a rotating tank, Phys. Fluids 2005;17(9):095105.

[60] van Bokhoven L J A, Cambon C, Liechtenstein L, Godeferd F S and Clercx H J H, Refined vorticity statistics of decaying rotating three-dimensional turbulence. J Turbulence 2008;9:115-127.

[61] Teitelbaum T and Mininni P D, The decay of turbulence in rotating flows, Phys. Fluids 2011;23(6):065105.

[62] Vorobev A, Zikanov O, Davidson P A and Knaepen B. Anisotropy of magnetohydrodynamic turbulence at low magnetic reynolds number, Phys. Fluids 2005;17(12):125105.

[63] Reddy K S and Verma M K. Strong anisotropy in quasi- static magnetohydrodynamic turbulence for high interaction parameters Phys. Fluids 2014;26(2):025109.

[64] Kolesnikov Y B and Tsinober A B. Experimental investigation of two-dimensional turbulence behind a grid. Fluid Dyn. 1976;9(4):621-624.

[65] Hossain M. Inverse energy cascades in three-dimensional turbulence. Phys. Fluids B 1991;3(3):511-514.

[66] Verma M K and Reddy K S. Modeling quasi-static magnetohydrodynamic turbulence with variable energy flux. Phys. Fluids 2015;27(2):025114.

[67] Favier B, Godeferd F S, Cambon C, Delache A and Bos W J T. Quasi-static magnetohydrodynamic turbulence at high Reynolds number. J. Fluid Mech. 2011;681:434-461.

[68] Mininni P D and Pouquet A. Helicity cascades in rotating turbulence. Phys. Rev. E 2009;79(2):026304. 